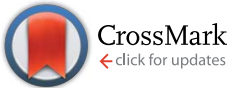

Cite this: Soft Matter, 2015, 11, 2530

Received 12th January 2015 Accepted 23rd February 2015

DOI: $10.1039 / \mathrm{c} 5 \mathrm{sm} 00084 \mathrm{j}$

www.rsc.org/softmatter

\section{Liquid marbles: topical context within soft matter and recent progress}

\author{
G. McHale*a and M. I. Newton ${ }^{\mathrm{b}}$
}

The study of particle stabilized interfaces has a long history in terms of emulsions, foams and related dry powders. The same underlying interfacial energy principles also allow hydrophobic particles to encapsulate individual droplets into a stable form as individual macroscopic objects, which have recently been called "Liquid Marbles". Here we discuss conceptual similarities to superhydrophobic surfaces, capillary origami, slippery liquids-infused porous surfaces (SLIPS) and Leidenfrost droplets. We provide a review of recent progress on liquid marbles, since our earlier Emerging Area article (Soft Matter, 2011, 7 , 5473-5481), and speculate on possible future directions from new liquid-infused liquid marbles to microarray applications. We highlight a range of properties of liquid marbles and describe applications including detecting changes in physical properties (e.g. $\mathrm{pH}$, UV, NIR, temperature), use for gas sensing, synthesis of compounds/composites, blood typing and cell culture.

\section{Introduction}

It may seem intuitively obvious that a solid which is described as "hydrophobic" would have water fearing properties and so will necessarily repel water. However, when Aussillous and Quéré rolled a droplet of water across hydrophobic grains of pollen and those grains adhered strongly to the droplet, encasing it in

${ }^{a}$ Smart Materials \& Surfaces Laboratory, Faculty of Engineering \& Environment, Northumbria University, Ellison Place, Newcastle upon Tyne, NE1 8ST, UK. E-mail: glen.mchale@northumbria.ac.uk; Tel: +44 (0)1912273660

${ }^{b}$ School of Science and Technology, Nottingham Trent University, Clifton Lane, Nottingham NG11 8NS, UK

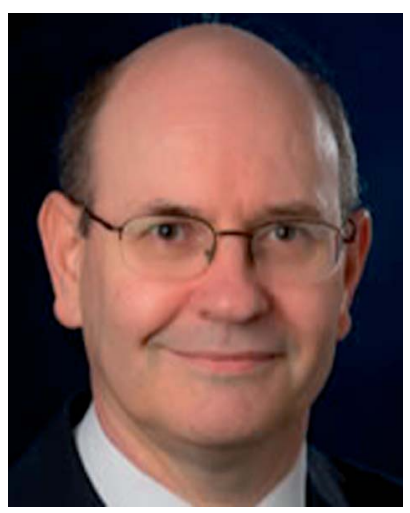

Professor Glen McHale received a BSc (Hons) degree in Mathematical Physics in 1983 and a PhD in 1986 in Applied Mathematics from the University of Nottingham, UK. Subsequently, he was a research assistant in theoretical physics at the University of Nottingham, a Royal Society European Research Fellow at the Université de Pierre et Marie Curie, and Professor of Applied \& Materials Physics at Nottingham Trent University. Since joining the University of Northumbria at Newcastle in 2012, his research has focused on the physics of static and dynamic wetting, superhydrophobic surfaces, dielectrowetting and drag reduction. a layer of powder and converting it into a complete non-wetting soft object, a "Liquid Marble" (Fig. 1a and b), they demonstrated how such intuition can be wrong. ${ }^{1,2}$ A simple calculation confirms that replacing a portion of a droplet's liquid-vapour interface by a solid-liquid interface when a smooth spherical particle attaches will always lower the surface free energy irrespective of the surface chemistry. ${ }^{3}$ Both hydrophobic and hydrophilic particles (or grains), where the distinction is whether the equilibrium contact angle, $\theta_{\mathrm{e}}$, defined by Young's Law on a smooth flat surface of the same material is above or below $90^{\circ}$, will adhere to the surface of a droplet of water. When the particles are smooth and spherical, this distinction exhibits itself as the geometric property of whether the particles have

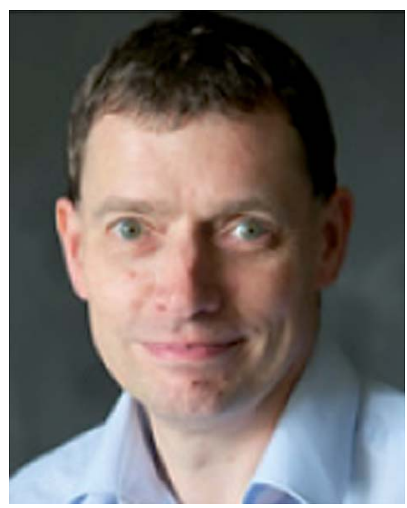

Dr Michael I. Newton received a BSc (Hons) degree in Physics in 1983, an MSc in Modern Electronics and a PhD in Semiconductor Physics from the University of Nottingham, UK. Subsequently he joined Nottingham Trent University and is currently a Reader in Experimental Physics. His research interests are focused on the development of instrumentation and surface patterning techniques for wetting processes on solid surfaces and for NMR-based sensors. His review of superhydrophobic surfaces, published in Soft Matter, is one of the most highly cited articles in the field. 

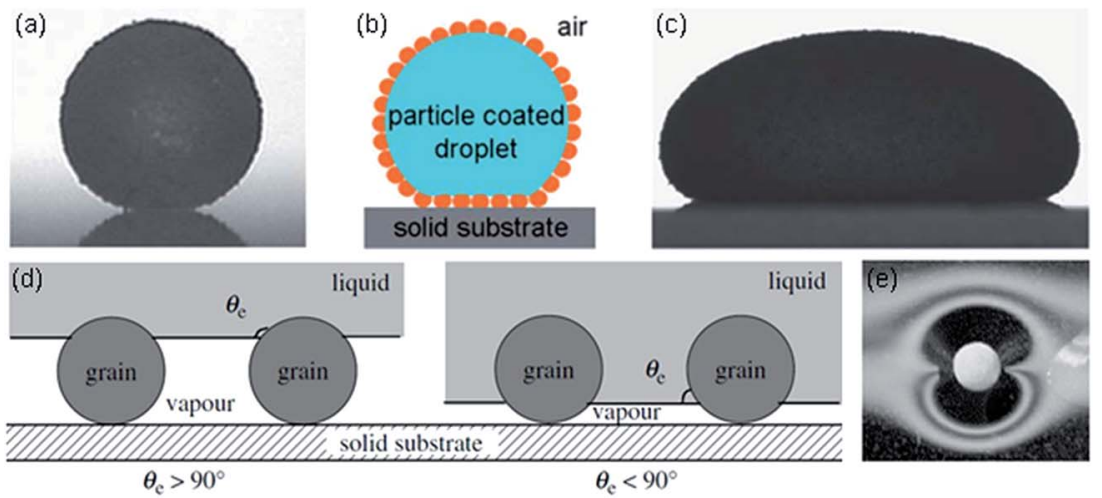

Fig. 1 (a) Liquid marble in air (radius $\sim 0.7 \mathrm{~mm}$ ), (b) schematic of encapsulation by hydrophobic particles, (c) particle encapsulated liquid puddle in air, (d) meniscus position of core liquid on particles according to hydrophobic or hydrophilic contact angle, (e) liquid marble floating on water $((\mathrm{a}-\mathrm{c})$ adapted from ref. 4; with permission from The Royal Society of Chemistry. (d and e) adapted from ref. 2; Copyright@ 2006 The Royal Society).

more than half their shape outside the droplet or inside the droplet (Fig. 1d). The particles that are most strongly bound to the surface of water are those whose surface chemistry lies on the transition between hydrophobic and hydrophilic, i.e. $\theta_{\mathrm{e}}=$ $90^{\circ}$. All liquid marbles encapsulate a liquid, converting what would have been contact between the liquid and a solid substrate into a solid-solid contact. Some liquid marbles go further and allow the encapsulated liquid to also remain separated from contact with a liquid substrate (Fig. 1e). Whilst these ideas are phrased in terms of hydrophilic and hydrophobic properties of solid particles and their interaction with droplets of water, the same concepts apply to oleophilic and oleophobic particles and their interaction with droplets of liquid. Moreover, as the volume of liquid increases the surface free energy considerations for adhesion of particles to the liquid surface remains valid although the thickness of the soft object formed is limited to twice the capillary length, $\kappa^{-1}$, so that large area particle-coated puddles are created rather than spherical marbles (Fig. 1c).

In an earlier report, we discussed liquid marbles as an emerging area and highlighted natural examples from the waxcovered liquid spheres used as a waste disposal system by Galldwelling aphids ${ }^{5}$ to the assemblies of liquid marbles created by wet granulation of hydrophobic powder. ${ }^{4}$ We briefly reviewed the properties of liquid marbles - properties and mathematical foundations which also been reviewed by others. ${ }^{2,6-9}$ However, our focus was on the conceptual basis of single macroscopic liquid marbles, and their emerging applications, and how those concepts bridged across to those of particle stabilised (Pickering) emulsions and the related field of dry water. ${ }^{10}$ In the present article, we provide an insight into how the field relates to other topical areas of Soft Matter, review progress in the field in the period 2011-2014 since our previous article and discuss prospects for the future, including suggestions for new directions.

In Section 2, we provide a conceptual framework for how liquid marble research might relate to superhydrophobic surfaces,${ }^{11}$ capillary origami ${ }^{12}$ Slippery-Liquid Infused Porous Surfaces (SLIPS) ${ }^{13}$ and the Leidenfrost effect. ${ }^{14}$ In Section 3, we address heat and mass transfer (evaporation/drying, condensation and freezing) including whether a liquid marble must necessarily evaporate more slowly than a droplet. This leads on in Section 4 to questions relating to the types of particles used and the liquids they encapsulate, and in Section 5 to consideration of the particle packing phases and shaping, setting and properties of the shell. In Section 6 we discuss the developing applications of liquid marbles as stimuli responsive objects, sensors and miniature chemical and biological reactors. We therefore aspire to provide a contextual and future directions review which builds upon and complements the principles and applications report of our previous Emerging Area article. ${ }^{4}$ Taken together these two articles provide an overview of the field up to the end of 2014.

\section{Topical context}

\subsection{Superhydrophobic (and superamphiphobic) surfaces}

A common method to alter the wetting of a solid surface is to use surface topography, at nano- and/or microscale, to amplify the natural wetting tendencies originating from the surface chemistry. ${ }^{15,16}$ One of the simplest models for the Cassie-Baxter state uses a cartoon of regular hydrophobic pillars. ${ }^{17}$ When a droplet of water contacts this surface it skates across it and contacts only the tops of the pillars. In a similar manner to the attachment of a particle to a droplet to form a liquid marble, there is a lowering of surface free energy when a portion of the droplet's liquid-vapour interface is replaced by a liquid-solid interface at the top of a pillar. The key difference is that the pillars are held in place by the underlying substrate and so the droplet shape is forced to conform to the pillar-adorned substrate (Fig. 2a). Such a droplet has reduced contact area with the solid compared to a smooth solid surface, but it nonetheless has a finite contact area and some residual adhesion to the substrate. To convert this cartoon to a liquid marble, we imagine a plane of structural weakness along the base of the pillar so that the force of the surface tension, $\gamma_{\mathrm{LV}}$, seeking to cause the droplet to ball-up into a sphere detaches each pillar (or where the weak plane is broken by means other than surface 
$\begin{array}{ll}\text { (a) Texture on superhydrophobic surface } & \text { (b) Texture conformed to liquid surface }\end{array}$

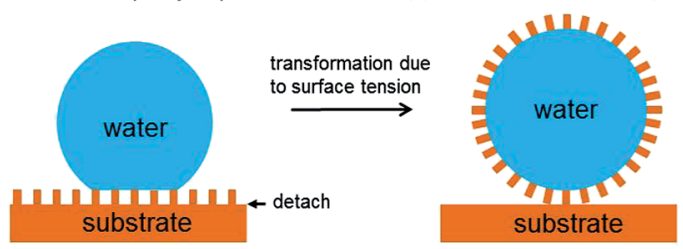

(c) Janus re-entrant pillar textured surface

(d) Pillars conformed to liquid surface

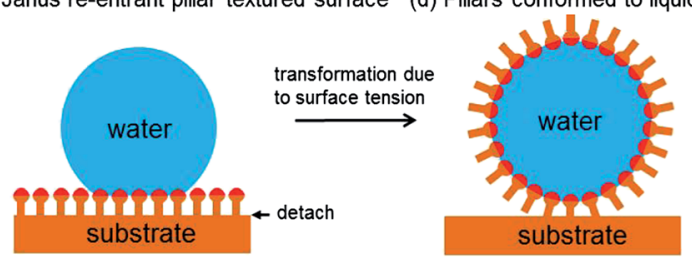

Fig. 2 (a) Droplet resting on pillars of a superhydrophobic surface, (b) detachment and self-assembly into conformal surface texture, (c) droplet resting in "ball-and-stick" Janus re-entrant curvature features, (d) liquid marble formed from Janus "ball-and-stick" structure.

tension after droplet contact). ${ }^{18}$ Thus, the pillars which commenced their lives as the topography of the substrate are converted into the particles that form the shell of the liquid marble (Fig. 2b).

Such a conceptual picture is not limited to surfaces possessing simple rectangular or circular cross-sectional pillars, but can be applied to surface features of any shape, such as spherical particles or "ball-on-stick" shapes possessing reentrant curvature (Fig. 2c). This would allow droplets of water (or other liquids) to become decorated in designer shaped particles initially seeded in the pattern of the features on the original substrate. Moreover, the shapes of each pillar could be different or the surface chemistry of the upper part of a pillar could be hydrophilic (creating "Janus" pillars) to control the orientation of the particles forming the shell of the liquid marble (Fig. 2c and d).

\subsection{Capillary origami}

The ability of a solid substrate to support a droplet of a liquid without itself deforming depends on its bending rigidity, $\kappa_{\mathrm{b}}$. Since the bending rigidity of a solid elastic plate scales with the cube of its thickness, a droplet can bend and wrap a membranelike solid film providing its radius is larger than the elastocapillary length, $L_{\mathrm{EC}}=\left(\kappa_{\mathrm{b}} / \gamma_{\mathrm{LV}}\right)^{1 / 2} \cdot{ }^{12,19}$ This ability of a droplet of water to wrap itself in a hydrophilic or hydrophobic solid film is another example of the lowering of surface free energy when a portion of the droplet's liquid-vapour interface is replaced by a liquid-solid interface. ${ }^{20,21}$ This is a tendency that is difficult although not impossible to suppress provided there is some degree of rigidity and superhydrophobic surface structure can be incorporated. ${ }^{22}$ The adhesion between water and a hydrophobic solid was vividly demonstrated in practice by Gao and McCarthy when they touched a thin film of Teflon with a droplet and the (hydrophobic) Teflon wrapped itself around the droplet. $^{23}$ The conceptual similarity to liquid marbles is that one can imagine a solid with low bending rigidity as a collection of particles with weak links ${ }^{\mathbf{2 0 , 2 1}}$ - pictorially one can imagine a net of knots where each knot is a particle. Thus, contact of a droplet to such a bed of hydrophobic particles will result in a droplet wrapping response as the imaginary net is forced to conform to the shape of the droplet.

The idea of structuring a substrate as if it were a net of loosely connected particles may offer the opportunity of new types of liquid marbles. When the loose connections between particles vanish completely we return to the usual liquid marble case where it can be formed by rolling a droplet across a bed of loose hydrophobic particles. The idea of a net also leads naturally to the question of what determines the strength of the connections between the particles when assembled into the shell of a liquid marble and whether those connections are liquid-like or solid-like in character as discussed in Section 5 in terms of granular rafts, capillary attraction, the Cheerio's effect and armoured droplets.

\subsection{Slippery liquid-infused porous surfaces (SLIPS)}

Superhydrophobic surfaces supporting droplets in the CassieBaxter state are only one way of using topographic structure (or texture) to create a surface that is slippery to water. To create a liquid shedding surface the necessary condition is that the contact angle hysteresis should be small. A high static contact angle surface which possesses high contact angle hysteresis will not easily shed droplets. In contrast, a low static contact angle surface with vanishing hysteresis will easily shed droplets. This has led to a recent focus in the literature on textured surfaces where the additional solid surface area leads to hemi-wicking of a non-volatile immiscible lubricating liquid (lubricant) into the surface, a so-called SLIPS surface. ${ }^{13}$ In the ideal case, the lubricant hemi-wicks into the texture and spreads across the tops of the surface texture features. For maximum slippery effect a complete layer of lubricant should be maintained between the solid and any droplet deposited on the surface (Fig. 3a). It is also possible with a SLIPS surface for the lubricant to spread as a thin film across the droplet surface (liquidvapour interface) thereby cloaking it from the vapour phase (Fig. 3b). ${ }^{24}$

The concept of a SLIPS surface raises interesting questions when the shell of a liquid marble is regarded as similar to the texture on a superhydrophobic surface, but conformed to the shape of the droplet. One can imagine a liquid marble whereby a lubricant is hemi-wicked and locked into the shell formed by the particles of the liquid marble. This would create a liquid droplet with a conformable SLIPS coating; Fig. 3c gives one possible example based on Fig. 2d. Whilst such a SLIPS coating would not be needed to make a liquid marble mobile on a solid surface, such a Liquid-Infused Shell Liquid Marble could be expected to have a reduced/vanishing rate of evaporation of the core liquid and modified impact and deformation properties. Such an object could also be viewed as a particle-stabilized multiphase compound droplet or, when immersed in another liquid, it could be regarded as a new type of particle-liquid stabilized interface. If created in large numbers, it could 
(a) Droplet on a SLIPS surface

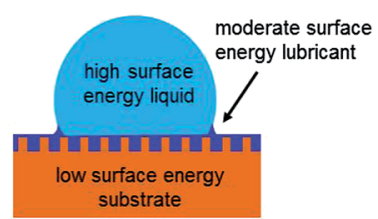

(b) Cloaked droplet on a SLIPS surface

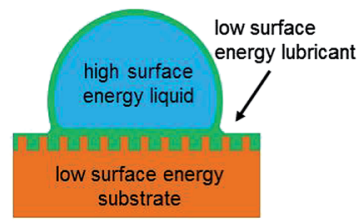

(c) SLIPS liquid marble

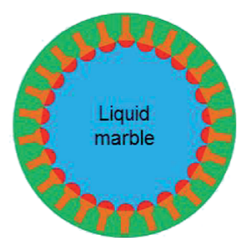

Fig. 3 (a) Droplet on a SLIPS surface formed by impregnating a texture with a lubricating liquid. (b) Droplet with a cloaking film of lubricating fluid from the SLIPS surface. (c) Liquid impregnating the surface texture of a liquid marble ( $(a$ and $b)$ adapted from ref. 24 with permission from The Royal Society of Chemistry).

resemble a new type of Pickering emulsion, including a type of dry water. ${ }^{10}$

\subsection{Leidenfrost droplets}

In the case of a superhydrophobic surface a droplet retains contact with a small, but finite, fraction of solid surface area, $\varphi_{\mathrm{s}}$. This has consequences for droplet adhesion, droplet mobility and heat transfer between the substrate and the droplet. Changes in heat transfer alter the modes of evaporation, and icing and condensation properties. An ideal superhydrophobic surface would be one for which the solid surface fraction vanishes, i.e. $\varphi_{\mathrm{s}} \rightarrow 0$, causing perfect non-wetting. Such a situation occurs when a droplet of water is brought into contact with an extremely hot substrate, above the Leidenfrost temperature, resulting in an instantaneous vaporization of a layer of water. ${ }^{1425}$ The vapour created forms a cushion that reduces heat transfer from the substrate and adhesion and friction between the droplet and the substrate (Fig. 4a). Conceptualizing a liquid marble as a system with the superhydrophobic texture, that is normally rigidly part of the substrate, conformed to the droplet focuses on the solid texture rather than the gas within the texture. Conceptualizing a liquid marble as a non-wetting droplet isolated from the substrate by a gas layer creates an analogy to a Leidenfrost droplet. These complementary views suggest a liquid marble might have some properties intermediate between a droplet on a superhydrophobic surface and a Leidenfrost droplet.

In terms of droplet mobility a liquid marble has low adhesion and high mobility because of the transformation of the liquid-solid substrate contact into a solid-solid substrate contact. Although it has high mobility, it is not the same as a droplet levitated on a vapour cushion where the residual
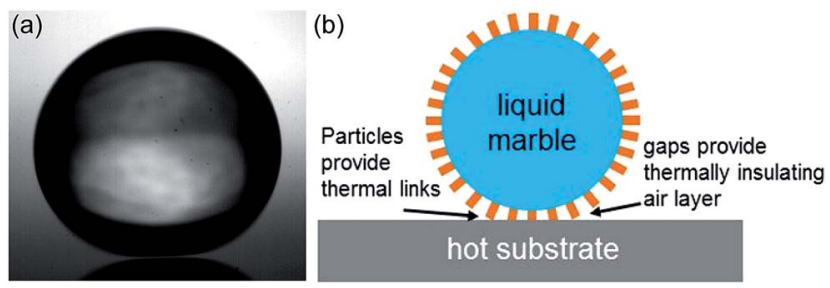

Fig. 4 (a) Leidenfrost droplet levitated on a thermally insulating vapour cushion. (b) Liquid marble viewed as a levitated droplet with both an insulating gap and thermal links to the substrate. friction is caused by viscous losses in the vapour flow, which itself can be manipulated by structuring the substrate. In terms of heat transfer from the substrate a liquid marble may have some similarities to a Leidenfrost droplet or to the superhydrophobic surface. If we consider particles in a shell as providing a physical spacing to lift the core liquid above the substrate then we can imagine an insulating vapour layer (Fig. 4b). However, there also remains direct thermal contact with the substrate via the droplet conformed texture of the surface shell and this is similar to a superhydrophobic surface. The particle size and arrangement to form the shell, and their thermal properties can be expected to influence heat transfer. Evaporation of the liquid marble will be influenced by evaporation through the shell and heat transfer from the substrate conducted through the shell.

\section{Heat and mass transfer}

\subsection{Evaporation and drying}

In our previous article ${ }^{4}$ we reviewed studies of evaporation of liquid marbles, which reported reduced evaporation rates, selfcoating effects and crumpling and buckling of the shell in the end stages. More recently, Doganci et al. has suggested that using low concentrations of water soluble surfactant (aqueous sodium dodecyl sulphate, SDS) in graphite micropowder liquid marbles can increase the time to buckling. ${ }^{26}$ This is due to the increased hydrophilicity of the SDS adsorbed graphite particles enabling more dense packing and the exclusion of air pockets from the graphite powder layer at the air-liquid interface. Erbil has also comprehensively reviewed evaporation of pure liquid and spherical suspended droplets, including recent work on droplets evaporating from superhydrophobic surfaces and evaporating liquid marbles. ${ }^{27}$ He noted that the lifetime of a liquid marble depends on the size and type of hydrophobic powder and also the liquid used to form it. Graphite marbles were noted as having twice the lifetime of pure droplets whilst PTFE marbles reduced evaporation rate by $25-45 \%$ compared to droplets. Moreover, the higher the relative humidity, the more effective the liquid marble's shell in retarding evaporation as might be intuitively anticipated. However, the counter-intuitive result that a liquid marble can increase the rate of drying compared to a droplet of the same volume provided the shell is in the form of a monolayer of particles rather than a multilayer aggregate has also been reported. ${ }^{28}$ The two principles 
underlying this are that (i) a monolayer of particles does not modify the average diffusion flux over the interface and so does not significantly reduce the water loss rate from the surface, and (ii) a close packed particle layer maintains a constant surface area during the buckling and crumpling phase.

In these previous studies of evaporation the process is a diffusion limited one in which the relative humidity controls the gradient in vapour concentration between the liquid marble and the surroundings. With a heated substrate the lifetime of a droplet decreases as substrate temperature increases until the substrate reaches the Leidenfrost transition temperature $\left(\sim 200{ }^{\circ} \mathrm{C}\right)$ whereupon droplet lifetime extends considerably due to the creation of an insulating layer of vapour. Aberle et al. conducted Leidenfrost-type superheat experiments on liquid marbles using a temperature range $100{ }^{\circ} \mathrm{C}$ to $465{ }^{\circ} \mathrm{C}$ and shells formed using 10-20 $\mu \mathrm{m}$ diameter graphite particles. ${ }^{29}$ For their liquid marbles they observed similar long lifetime behaviour to that of a Leidenfrost droplet, but across the full temperature range and without any equivalent to a Leidenfrost transition temperature. In their case, the size of particles used gives a separation of the core liquid from the hot substrate in the $\sim 15-$ $30 \mu \mathrm{m}$ range similar to the $\sim 10-100 \mu \mathrm{m}$ insulating vapour layer thickness reported for Leidenfrost droplets. The suggestion in these experiments is that the (insulating) particles provide the spacing of the liquid from the substrate and the air in gaps between particles is the insulating vapour. For liquid marbles one might expect different results depending on whether particles are thermally conductive or sub-micron and uniform in size, and whether the shell is an aggregate of multiple layers. Interestingly, if we consider the view point of a liquid marble as a droplet with superhydrophobic texture conformed to its surface and providing a stable insulating layer of air, there may be a similarity in the liquid marble results to the reports of suppression of the Leidenfrost transition temperature for Leidenfrost droplets on hot superhydrophobic surfaces. ${ }^{30}$ In these experiments, a textured superhydrophobic surface was shown to eliminate the collapse of the vapour film below a droplet, thus making it possible to maintain a stable vapour film at all temperatures above the boiling point of the liquid.

\subsection{Condensation}

The limited studies relating to liquid marbles and condensation have focused on producing liquid marbles via condensation as an alternative mechanism to deposition of a droplet on a hydrophobic particle bed or the use of an industrial-type mixer. ${ }^{31}$ Rykaczewski et al. showed microscale liquid marbles (maximal diameters $\sim 300-400 \mu \mathrm{m}$ ) can be formed through selfassembly during water condensation on a superhydrophobic surface covered with a loose layer of hydrophobic nanoparticles. $^{32}$ They noted that formation of their liquid marbles was driven by multiple coalescence events between partially covered droplets and that surface flows, possibly driven by the Marangoni effect, aided nanoparticle film redistribution on the liquid marble. Bhosale and Panchagnula also studied the potential for large scale production of well-defined size liquid marbles via a condensation-driven liquid marble formation process. ${ }^{33}$ They referred to their method as "sweating" reflecting the use of an evaporation-condensation process. The study included water, glycerol and ethylene glycol to span an order of magnitude in viscosity, and hydrophobic fumed silica nanoparticulate (nHMDS) and microparticulate polytetrafluoroethylene ( $\mu$ PTFE), although the process was reported as most repeatable with nHMDS. They concluded that the primary mechanism causing the formation of liquid marbles was droplet nucleation followed by condensation-driven growth with drop coalescence a secondary mechanism with potentially high growth rates, but causing variability in the size distribution. In these experiments we see the driving effect of the lowering of surface free energy when hydrophobic particles adhere to a liquid-vapour interface and the freedom for particles forming a substrate or bulk material to be conformed to the surface of a droplet.

\subsection{Freezing}

Although Rykaczewski et al. briefly reported on the freezing of their microscale liquid marbles ${ }^{32}$ a more extensive study for macroscopic liquid marbles was undertaken by Hashmi et al. ${ }^{34}$ Their study used liquid marbles formed from deionised water and lycopodium particles on a silicon wafer maintained at $-8{ }^{\circ} \mathrm{C}$. They reported a series of shape changes with a bulging near the solid surface causing a bellshape and then, usually, evolution to a flying saucer type shape, as the freezing front moved gradually from the substrate towards the top of the liquid marble. These shape changes appeared different to those occurring for a droplet freezing on a cooled superhydrophobic surface. Most recently, the freezing of liquid marbles with fumed silica nanoparticle have been shown to exhibit tuneable shape transformations which are dependent on the hydrophobicity of the nanoparticles. ${ }^{35}$ For highly hydrophobic particles frozen shapes possessing a pointed protrusion similar to those occurring for droplets on superhydrophobic surfaces were observed, whilst for less hydrophobic particles a lateral expanded flying saucer-shaped morphology occurred (Fig. 5a and b). It appears that different hydrophobicities cause different heterogeneous nucleation sites owing to the different positions of the particles at the air-water interface (Fig. 5c). For more hydrophilic particles ice embryos tend to form in the concave cavities between particles whereas for more hydrophobic particles nucleation occurs on the air exposed convex surfaces of the particles.

\section{Types of particles and liquids}

\subsection{Particles for encapsulating low surface tension liquids}

The original studies of liquid marbles tended to use water or glycerol-water mixtures as the core liquid. By 2007 this had already been extended to ionic liquids ${ }^{\mathbf{3 6}}$ (see also reports of “ $d r y$ ionic liquids"37) and by 2010 a wide range of other liquids including organic solvents with surface tensions as low as $20.1 \mathrm{mN} \mathrm{m}^{-1}$. ${ }^{4}$ Similar ability to encapsulate liquids from water to organic liquids, including volatile liquids, such as toluene, chloroform and tetrachloromethane have been reported for 

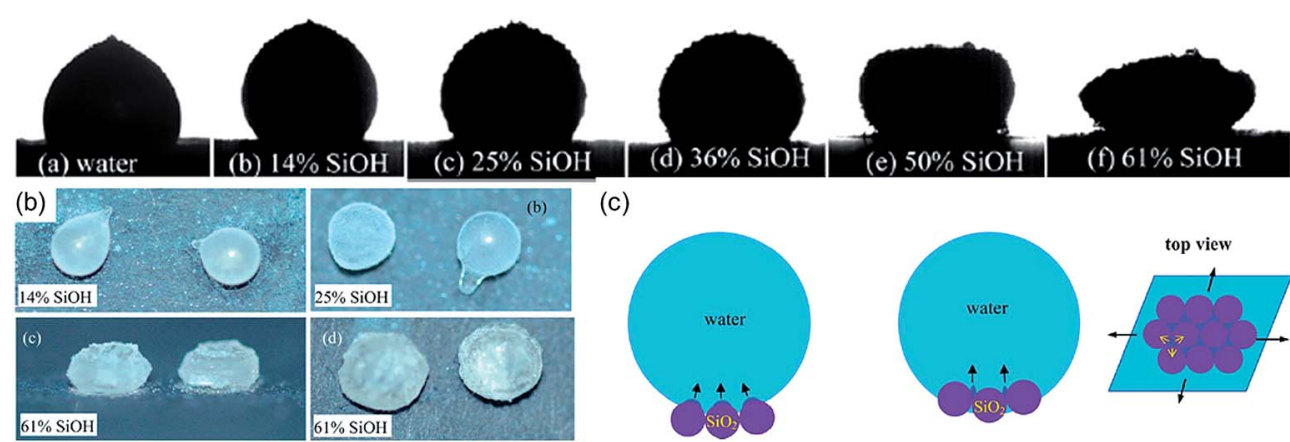

(c)

Fig. 5 (a) Final solidified liquid marble shapes from being placed on a cold plate: pointed protrusion to flying saucer morphology. (b) Final shapes when solidified in a freezer. (c) Postulated freezing process with heterogeneous nucleation from convex particle surface (hydrophobic particle case) to cavity embryos (hydrophilic particle case) (reproduced from ref. 35. with permission from The Royal Society of Chemistry).

superoleophobic perfluorooctanoic acid (PFOA) modified $\mathrm{TiO}_{2}$ nanoparticles. ${ }^{38}$ Binks and Tyowua also reported systematic studies of the behaviour of fumed silica particles coated to different extents with a perfluoro-alkoxysilane in mixtures of air and liquids ranging from non-polar hydrocarbons and polar oils to glycerol to water. ${ }^{39}$ They discussed the transformation of the bulk material from oil-in-air liquid marbles to particle-stabilised air-in-oil foams as the contact angle of the particles with the air-liquid interface increases. In subsequent work it was shown that a series of platelet sericite particles coated to different extents with a fluorinating agent could be used to create oil liquid marbles, dry oil powders and stable oil foams. ${ }^{40}$ Systematic studies of controlled silanization of silica nanoparticles for use with methanol-water mixtures have also been reported ${ }^{41}$ and the use of propylene glycol with particle-stabilized emulsions of paraffin oil and water. ${ }^{42}$ Dyab and Al-Haque also prepared non-aqueous systems of emulsions, foams, liquid marbles and polymeric materials stabilised by either dichlorodimethylsilane (DCDMS)-modified amorphous silica or organo-modified Laponite clay nanoparticles. $^{43}$ Macroscopic water and water-glycerol liquid marbles can also be immersed in organic liquids and oils. ${ }^{44}$ Amongst particles able to encapsulate low surface tension liquids poly[2-(perfluorooctyl)ethyl acrylate] $\left(\mathrm{PFA}^{-\mathrm{C}_{8}}\right)$ microparticles $(\sim 1.4 \mu \mathrm{m})$ appears capable of very low surface tensions including methanol with $\gamma_{\mathrm{LV}}=9.8 \mathrm{mN} \mathrm{m}^{-1} .^{45}$ These authors (and others, e.g. Binks et al. ${ }^{39,42}$ ) have discussed the ability of particles to contain a given liquid in terms of the spreading coefficient for liquid-on-solid in air, $S_{\mathrm{S} / \mathrm{L}}$, and related it to the ideas of dispersive and polar components of the liquid and solid.

One way of characterising whether a liquid will penetrate a particle bed or not, and hence the potential for formation of a liquid marble, is to use the Molarity of Ethanol Droplet (MED) test (also referred to as the \%ethanol test). This test examines whether droplets with increasing concentrations of ethanol in water, and hence successively lower surface tension solutions, penetrate into the particle bed. This is related to the concept of critical surface tension and is used extensively in studies of soil water repellency (see references in Hamlett et al. ${ }^{46}$ ). Bormashenko et al. studied liquid marbles formed from droplets of water-ethanol solutions of various concentrations coated with polytetrafluoroethylene, polyvinylidene fluoride and polyethylene and reported that there is a critical surface tension of the water-ethanol solutions for the formation of liquid marbles. ${ }^{47}$ Whitby et al. also studied particle coating of alcohol-water drops placed onto porous beds of hydrophobic coarse glass spheres (spherical, 90-106 $\mu \mathrm{m}$ ), coal dust (angular shape, 90-106 $\mu \mathrm{m}$ ) and molybdenite powder (angular shape, 1-20 $\mu \mathrm{m}$ ), thereby varying particle bed packing geometries. ${ }^{48,49}$

Whilst the critical surface tension as a limit to liquid marble formation might be a natural expectation for droplets deposited on a multi-layer bed of particles, it may not necessarily represent the lowest surface tension liquid for which a given set of particle can encapsulate it as a liquid marble. When a droplet is placed on a multilayer bed of particles, the wetting transition may depend solely on the contact angle of the liquid with the particles provided the droplet is small and the particles are relatively non-wetting. However, for more wetting liquids imbibition into the particle bed will occur when the liquid advances sufficiently into the first layer of particles such that the meniscus touches the second layer of particles. ${ }^{46,50}$ Thus, although it may not be possible to form a liquid marble by depositing it onto a thin (multi-layer) film of a powder, it could still be possible to create one by depositing the same particles onto a droplet using a different technique. One might expect this latter situation to be predicted using a spreading coefficient approach or by the condition $\theta_{\mathrm{e}}>0^{\circ}$. We would expect such a liquid marble could be deposited onto a flat surface, but would rupture if placed on a powder. This also highlights that we can expect the stability of a liquid marble to depend upon the length scales of roughness of the solid surface it is placed upon. If surface features can project between the particles in the shell, they could induce wicking of the liquid from the core of the liquid marble leading to rupture. 


\subsection{Magnetic/magnetizable particles}

Over the last four years there have been numerous reports relating to magnetic particles for use in the shells of liquid marbles motivated by the potential for external actuation and control. The review of Tippkötter et al. focuses on the principles, synthesis and functionalization of magnetic particles for biotechnological applications motivated by their potential for target binding and selective separation from fermentation or reactions broths. ${ }^{51}$ Their review includes both non-magnetisable shells encapsulating liquids containing magnetic beads and synthesis of particles providing a magnetisable shell. Progress towards magnetically controlled liquid marble microfluidics has continued with Zhao et al. extending previous work to demonstrate optical probing of the absorbance curves of core solutions through gaps in superhydrophobic $\mathrm{Fe}_{3} \mathrm{O}_{4}$ nanoparticle magnetic shells that could be opened and closed reversibly. ${ }^{52}$ Magnetic nanoparticles with a UV triggered hydrophilic transition have been synthesized thus providing for a remotely triggered rupture of a liquid marble to complement magnetically actuated motion. ${ }^{53} \mathrm{Fe}_{2} \mathrm{O}_{3}$ nanoparticles have also been dispersed in the core ionic liquids of PTFE particle based liquid marbles to provide magnetic actuation and control. ${ }^{54}$

A flame synthesis method combining flame combustion, aerosol coating and surface functionalization into a continuous gas-phase process to create hydrophobic magnetic chain-like nanoparticles (HMCNPs) has been reported (Fig. 6a).$^{55}$ Liquid marbles with shells of these particles can be magnetically actuated, but are also reported to have robust mechanical properties with rapid self-recovery from being compressed flat (Fig. 6b). Related work showed the continuous flame synthesis strategy can be adopted for large-scale preparation of spherical double-faced $\gamma-\mathrm{Fe}_{2} \mathrm{O}_{3} \| \mathrm{SiO}_{2}$ nanohybrids and their in situ chemical selective bonding of 3-methacryloxypropyltrimethoxysilane. ${ }^{56}$ These modified double-faced nanohybrids are Janus particles and self-assemble at oil-water or air-water interfaces (Fig. 6c). Zhao et al. prepared large $(\sim 100 \mu \mathrm{m})$ amphiphilic multi-compartment particles incorporating magnetic nanoparticles using an on-demand emulsion approach which could incorporate up to four dispersed phases to provide, e.g. structural colour. ${ }^{57}$ Dual wettability was demonstrated to provide magnetically responsive multi-compartment Janus particles with potential for spectra to be used for encrypting information and bar-coding liquid marbles. At the larger (whole liquid marble) scale, multi-responsive Janus liquid marbles with magnetic actuation and the ability to be ruptured by exposure to infra-red or acid/base vapours have been reported. ${ }^{\mathbf{5 8}}$ These marbles used an infra-red sensitive wax/hydrophobic superparamagnetic $\left(\mathrm{Fe}_{3} \mathrm{O}_{4}\right)$ particle powder for a semi-shell of the marble and a $\mathrm{pH}$ responsive no-magnetic modified $\mathrm{TiO}_{2}$ powder for the other semi-shell of the liquid marble rather than Janus-particles.

In the context of whether ideas underlying SLIPS surfaces could be translated to liquid marbles, an interesting possibility is the use of ferro-fluid impregnated surfaces for active manipulation of droplets. Khalil et al. used a ferro-fluid as the lubricating fluid on a textured substrate, but when a water droplet contacted the surface it self-coated in a ferro-fluid cloak. ${ }^{59}$ Whilst this work did not explicitly address liquid marbles, it suggests that a ferro-fluid with suitably functionalised magnetic particles could be used to construct a magneticresponsive liquid marble that would also be encapsulated in an immiscible liquid.

\subsection{Shaped particles}

In Section 4.1 and 4.2, encapsulating particles included shapes beyond simple spherical, such as platelet shapes and chain-like assemblies. Shape of individual particles can influence wetting in a variety of ways including enhancing the intrinsic wettability or non-wettability originating from the surface chemistry, providing orientation preferences when adhering to a liquid-air interface and, for very high curvature of the particle surface, altering the spreading coefficient required for a particle to be engulfed by a liquid to above zero, e.g. see Quéré et al. ${ }^{60}$ Moreover, anisotropic particles with high aspect ratio can stabilize emulsions. Thus, shape at the individual particle level influences properties of liquid marbles due to both local topography and global geometry. The definition of shape can also be broadened to include whether a particle is hollow or not, e.g.
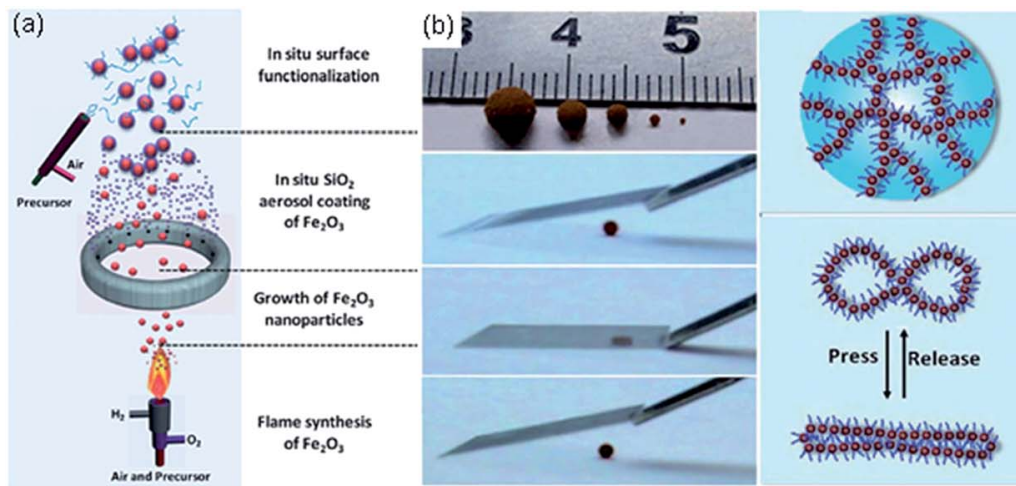

(c)

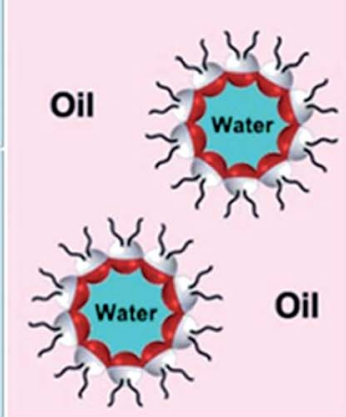

Fig. 6 (a) Flame synthesis of hydrophobic magnetic chain-like nanoparticles. (b) Rapid self-recovery from compression (reproduced from ref. 55. with permission from The Royal Society of Chemistry). (c) Double-faced nano-hybrids self-assemble at oil-water (and air-water) interfaces (reproduced from ref. 56. with permission from The Royal Society of Chemistry). 
Binks et al. ${ }^{61}$ or whether it has a pore structure. ${ }^{62}$ At the collective level of a group of particles at a liquid-vapour interface it also influences capillary interactions and packing, and so is likely to alter both the elastic and hard shell states of liquid marbles (see Section 5). A wide range of methods exist for preparing particles with different shapes. A recent example focused on producing complex particle shapes (beads, capsules and rods) through the formation of high internal phase emulsion droplets in a microfluidic channel (Fig. 7a). ${ }^{63}$

One method to achieve a simple shape change is to decorate a large particle with much smaller particles thereby creating a superhydrophobic particle surface structure. However, this requires the adhesion of a small hydrophobic particle to a larger particle. One approach used copper particles decorated with silver nanoparticles and studied the stability of the resulting liquid marbles as the weight of particles increased. ${ }^{65}$ Another approach for applications in wet environments used a core particle encapsulated in highly adhesive polydopamine which was then decorated with nanoparticles; ${ }^{66}$ see also Liang et al. ${ }^{67}$ As an example, carbonyl iron particles were used as the core material and superhydrophobic magnetic particles developed that possessed a water contact angle $\sim 160^{\circ}$, but with an oil contact $\sim 0^{\circ}$. When these particles were added to oil floating on water and a magnetic field applied to pull the magnetic particle-oil mixture below the surface and then released a water-immersed oil-marble formed spontaneously thereby demonstrating potential for oil-water separation and clean-up of oil.

Recent work using particle shape includes nano-rods, nanotubes, nano-fibers, nano-sheets and nano-belts. Nakai et al. used carbon nanotubes and fullerene $\left(\mathrm{C}_{60}\right)$ powders to create liquid marbles although their focus was not on shape, but the highly absorptive properties which allowed conversion of near infrared light into heat causing liquid marbles to rupture due to an external stimulus. ${ }^{68}$ The use of high aspect ratio inorganic halloysite nanotubes has also been reported to create liquid marbles with pincushion agglomerates providing superhydrophobic topography. ${ }^{69}$ Nanofibrillated cellulose (NFC) has been used to make superhydrophobic surfaces by spray coating and microparticles of NFC used to form liquid marbles. ${ }^{70,71}$ The use of electrospun nanofibers has been reported to increase the mechanical strength of liquid marbles and enable survival under high compression, immersion in silicone oil and vortex mixing. ${ }^{72}$ These authors classified their electrospun fiber mats into hydrophobic and superhydrophobic and noted that impact of droplets onto the mats only formed liquid marbles in the former case. Functionalised graphene nanosheets have been used for liquid marbles. ${ }^{73,74}$ Hydrophobic $\mathrm{Fe}_{3} \mathrm{O}_{4} / \mathrm{C}$ microsheets have also been used to achieve mechanically robust low evaporation rate liquid marbles which can be used as microreactors for the synthesis of graphene/Ag nanocomposites. ${ }^{75}$ Within the related field of Pickering emulsions it is interesting to note that surface and edge modified amphiphilic nanosheets (having both hydrophilic and hydrophobic properties), analogous to Janus particles and Gemini platelets, have been fabricated and used to stabilize oil-water interfaces. ${ }^{76}$ Titanate nanobelt (TNB) powders treated with different amount of fluoroalkylsilane (FAS) have been shown to possess different wetting states from relatively low to very high hydrophobicity and can self-assemble into a tightly cross-stacked layer at the air-water interface. ${ }^{64}$ To demonstrate the effect of hydrophobicity on the strength and flexibility of the TNB/FAS membrane they investigated its recovery from the insertion of a hydrophilic glass rod on hydrophobic, superhydrophobic and highly superhydrophobic particle rafts (Fig. 7b).

\subsection{Liquid metal marbles}

An emerging area of Soft Matter is the use of room temperature liquid metals that are deformable and shape-reconfigurable. ${ }^{77}$
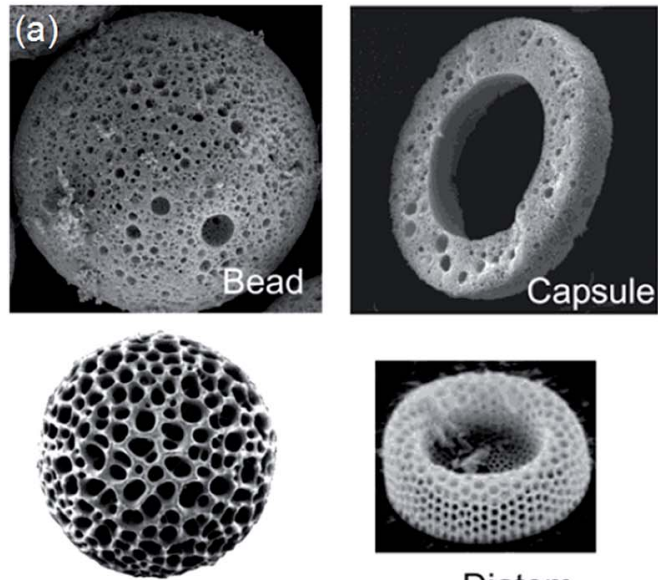

Radiolarian

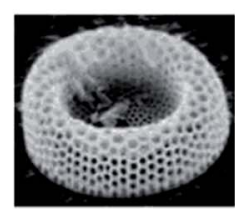

Diatom
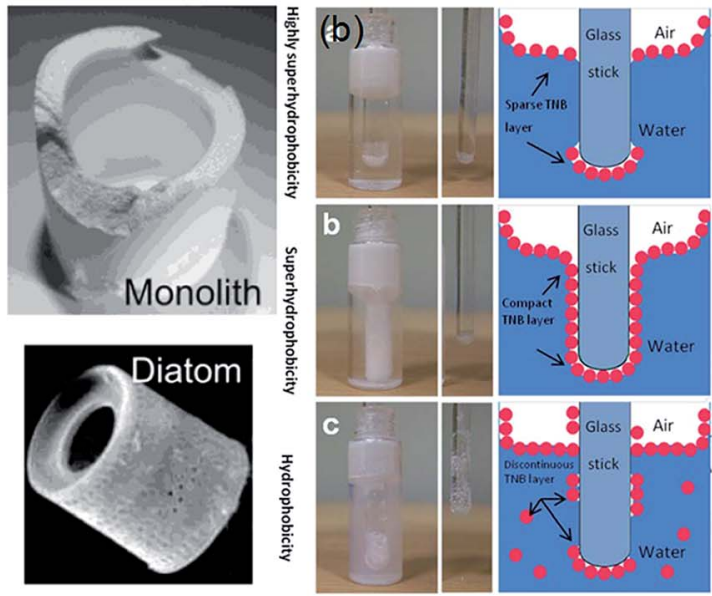

Fig. 7 (a) Examples of shaped particles for use with liquid marbles (reproduced from ref. 63. Copyright@ 2013 WILEY-VCH Verlag GmbH \& Co. KGaA, Weinheim). (b) Mechanical robustness of titanate nanobelt (TNB) powders under insertion of a hydrophilic glass rod into TNB layers at the water-air interface. Top-to-bottom: highly superhydrophobic with sparse TNB layer, superhydrophobic with compact TNB layer, and hydrophobic with discontinuous TNB layer (reproduced from ref. 64. with permission from The Royal Society of Chemistry). 
These have been used for soft electronics, antennas, 3D printed metal structures, and self-healing and stretchable wires (Fig. 8). Mercury is the best-known liquid metal, but it is toxic and its high surface tension tends to make it ball-up thereby making shape control difficult. Recent interest has therefore focused on the less toxic Gallium and its alloys and have used the native oxide layer to stabilize non-spherical shapes. ${ }^{78,79}$ Interestingly, control of the surface oxide layer allows the surface tension to be reversibly tuned from $500 \mathrm{mN} \mathrm{m}^{-1}$ to $\sim 0 \mathrm{mN} \mathrm{m}^{-1}$ thereby allowing shapes to be controllably re-configured. ${ }^{80}$ It has also been suggested that the ability to control the surface tension, and hence the wetting of a liquid metal, via an oxide layer means that surface oxides may be regarded as surfactants for metals.

Sivan et al. noted that liquid metals are corrosive and will dissolve and amalgamate with metal contacts. ${ }^{81}$ Moreover, the oxide layer on eutectic alloys of Gallium in air results in strong adhesion to glass and low surface energy polymers (such as polydimethylsiloxane). They studied liquid metal galinstan (a eutectic alloy of $\mathrm{Ga}$, In and $\mathrm{Sn}$ ) coated with $\mathrm{WO}_{3}$ nanoparticles in air and under water. They also reported that Teflon, polar insulators (e.g. $\mathrm{SiO}_{2}$ ), semiconductors (e.g. $\mathrm{CuO}, \mathrm{ZnO}, \mathrm{WO}_{3}$ ) and carbon nanotubes could all be used to encapsulate their liquid metal. They were able to roll, merge and split their marbles, suspend them on water and study impact. They also studied properties made possible by the high conductivity of the core liquid metal, including electrochemical properties and the use of semiconducting or insulating particles to create metal-semiconductor-metal junctions. One difference to the usual liquid marbles was that forming them in air led to non-uniform coating due to the surface oxide layer inhibiting particle rearrangement in the shell. However, the oxide layer can be reduced in a dilute $\mathrm{HCl}$ solution. Related work by the same group has demonstrated UV-induced photochemical actuation and electrochemical actuation of $\mathrm{WO}_{3}$ coated galinstan liquid metal marbles immersed in electrolyte solutions. ${ }^{\mathbf{8 2 , 8 3}}$ Combining the ideas of liquid metal marbles with the reversible tuning of the oxide layer reported in Khan et al. may provide further possibilities through switching and control of shape. ${ }^{80}$

\section{Types of shells}

\subsection{Phases of particle monolayers}

From the literature on heat and mass transfer, and mechanical robustness of liquid marbles it is clear that properties can be very different according to how a monolayer of particles is arranged and whether the shell is a multi-layer aggregate. Although this type of characterisation is absent from some of the literature a body of work has been building about the phases monolayers of particles can adopt on the liquid surface and how particle shells can be converted into different and more robust encapsulations.

One might envisage that the state of particles adhering to a liquid-vapour interface could undergo a series of phase transitions similar to Langmuir-Blodgett films as the surface areal density is increased. In such a view the particles could transition from a non-interacting gas to a liquid-like state and then to a solid-like state before collapsing. For a liquid marble, this suggests that for single layers of particles liquid-like and solidlike particle states will exist although one can expect more complex behaviour. For example, it is known that particles with the same hydrophobicity (or the same hydrophilicity) will have a net attractive force between themselves due to the relative signs of curvature of the liquid-vapour interface menisci at the particle surface, see e.g. Vella and Mahadevan, ${ }^{\mathbf{8 4}}$ and Kralchevsky and Nagayama. ${ }^{85}$ This leads to the simple rule that pairs of particles inducing menisci with like curvatures attract, whereas those inducing opposite curvatures repel. This concept can be expected to drive particles to form aggregates on the surface of a liquid marble rather than remain separated. It also leads to a simple question of whether liquid marbles can be fabricated which have hydrophilic particles controlling the separation between hydrophobic particles or whether the result would be liquid marbles with domains of separate hydrophilic and hydrophobic aggregates.

The confinement of particles on the surface of a liquid marble allows surface tension to provide a two-dimensional isotropic compressive stress and this can cause a jamming transition and shear-induced liquid-solid transitions leading to close-packed "armoured" droplets. ${ }^{86}$ This transition from
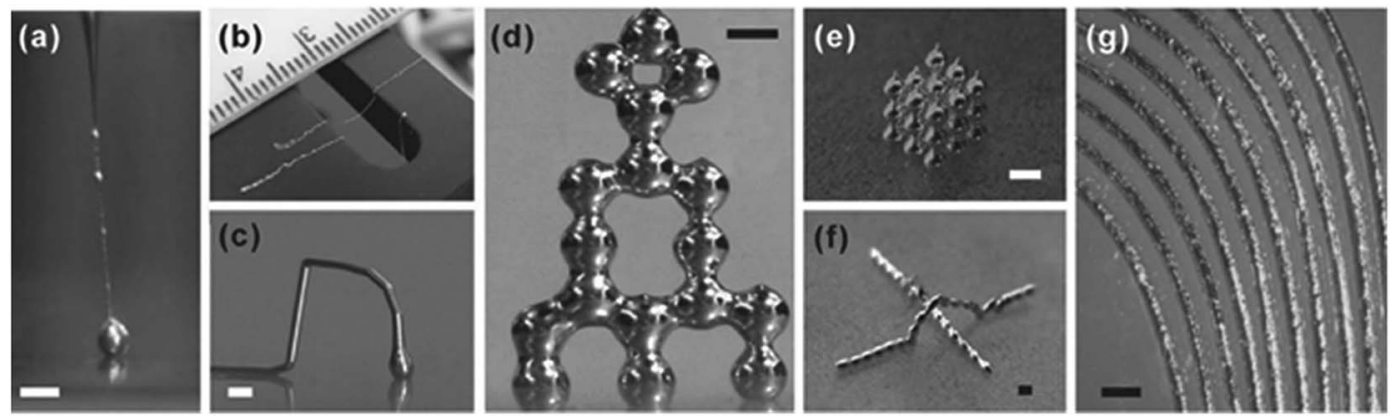

Fig. 8 Direct writing of liquid metal 3D structures. Free standing liquid metal structures that can be direct printed at room temperature. (a) Wire formed by ejection from a capillary. (b) Liquid wires suspended over a gap. (c) Free-standing liquid metal arch. (d) A tower of liquid metal droplets. (e) Stacked cubic array of droplets. (f) Liquid metal arch over a liquid metal wire. (g) Array of in-plane metal wires (reproduced from ref. 78. Copyright@ 2013 WILEY VCH Verlag GmbH \& Co. KGaA, Weinheim). 
capillary-like to jammed granular-like phases as particle surface fractions on liquid marbles increase has been discussed by Lagubeau et al. ${ }^{87}$ By following the shape and grain network organization of slowly evaporating liquid marbles they identified three shell phases: liquid-like $(\mathrm{L})$, soft-jammed solid $\left(\mathrm{S}_{1}\right)$ and hard-jammed solid $\left(\mathrm{S}_{2}\right)$. They suggested the L-phase represented the liquid marbles first reported by Aussillous \& Quéré ${ }^{1}$ with a cohesion-dominated loose structure stabilized by capillary interaction and having a shape determined by a Laplacetype law with a reduced surface tension. However, as the liquid marble evaporates a transition to a soft-jammed solid occurs where the concept of surface tension is misleading as global shear stress develops as the interface acquires rigidity. As evaporation continues the particle network undergoes a transition from a disordered to ordered state, characterised by a further jump in rigidity, prior to the onset of a wrinkled state. Using such jamming transitions it should be possible to create non-spherical armoured liquid marbles in a similar manner to non-spherical bubbles. ${ }^{88}$

\subsection{Effective surface tension and particle rafts}

Planchette et al. have considered the analogy of a particle-laden liquid-vapour interface with a surfactant-laden liquid-vapour interface noting similarities, such as the lowering of surface tension and macroscopic properties of buckling under compression and a subsequent bending energy, and dissimilarities, such as fracture/cracking and aging common to 2D granular systems. ${ }^{89}$ By considering the propagation of surface waves at a water-air interface covered with hydrophobic monodisperse particles they obtained values of a stretching modulus (i.e. effective surface tension) $\gamma_{\mathrm{e}}$ and bending stiffness. They observed no dependence of the effective surface tension on contact angle or particle diameter for the ranges they investigated. Their bending stiffness varied as the surface tension, $\gamma_{\mathrm{LV}}$, times the square of the particle diameter, $d$, and with little obvious dependence on contact angle. Their work built upon previous consideration of a particle raft which it was argued behaved approximately as an isotropic solid that could support anisotropic stresses and strains and whose elastic properties could be characterised in terms of a Young's modulus (with $\left.E=2.82 \gamma_{\mathrm{LV}} / d\right)$ and Poisson ratio. ${ }^{90}$ More complete references are provided by Dixit and Homsy in their consideration of interfacial elasticity on Landau-Levich flow. ${ }^{91}$

Experimentally liquid marbles appear well suited to experiments investigating changes in particle surface area coverage either by evaporation to increase packing fractions or by forced inflation/deflation through an attached syringe. Both evaporation and the latter approach using pendant drop liquid marbles were used with five hydrophobic and one hydrophilic powder to measure the effective surface tension, although the initial powder coatings where not homogeneous, hermetic (i.e. they displayed water clearings) or single layer. ${ }^{92}$ In these cases, the limiting case when inflating the liquid marbles tended to the surface tension of water as expected. As liquid marble size reduced, effective surface tension decreased inversely with surface area until a buckling transition occurred. Further studies using inflation/deflation of liquid marbles with carefully controlled monolayer systems, such as mono-disperse micronsize particles of poly(methylsilsesquioxane) which provide a particulate monolayer with a hexagonally close-packed (HCP) structure, ${ }^{93}$ could provide further insight. New approaches to the measurement of the effective surface tension of liquid marbles include a capillary rise method using insertion of a glass capillary tube into a liquid marble. ${ }^{\mathbf{9 4}}$ These results were discussed in terms of whether the particles on the surface formed a liquid-like state. A summary of different methods of measuring effective surface tension can be found in Cenzig and Erbil. ${ }^{95}$

An interesting variation on liquid marbles and particle stabilised emulsions is the phenomena of gravity-induced encapsulation of liquids by destabilization of granular rafts. ${ }^{96}$ In this work heavy particles sediment through a film of oil spread on water and form a close-packed capillary stabilised raft at the oilwater interface. An area of the raft can destabilise spontaneously or by being externally perturbed (e.g. by insertion of a rod) and it then sinks taking with it a particle coating and so forming one or more armoured oil-in-water droplets (Fig. 9). These authors suggested that such an approach could be used to remove a thin oil layer following a spill rather than using a skimmer method of remediation.

\subsection{Shaping and setting shells}

Liquid marbles can be created with different properties for different parts of their shell, such as with a Janus-type liquid marbles where one hemisphere is coated in carbon black (semiconductor) and the other in polytetrafluoroethylene

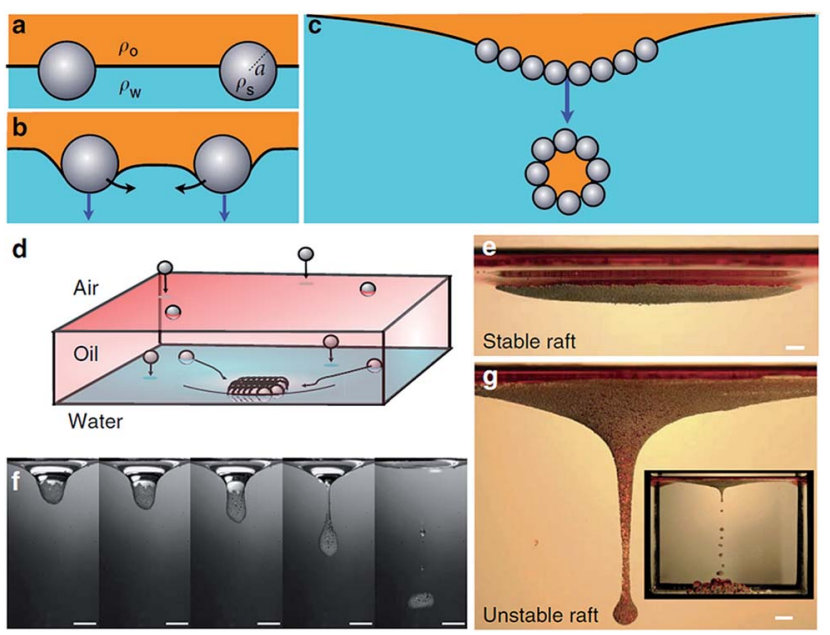

Fig. 9 Formation of armoured droplets from a raft of particles. (a)-(c) Particle interactions at an oil-water interface leading to a raft which destabilizes. (d) Experimental set-up with a lower phase of water onto which a layer of mineral oil is deposited. (e)-(g) Capillary raft floats at water-oil interface or destabilizes according to the number of particles in the raft. (f) Destabilization with encapsulation of a single droplet of oil. (g) Destabilization into a long jet of oil which subsequently breaks into multiple armoured droplets. (Reprinted by permission from Macmillan Publishers Ltd: Nature Communications ref. 96. Copyright@ 2013 Macmillan Publishers Limited). 
(dielectric), and this can allow orientation using an electric field. ${ }^{97}$ Bormashenko et al. has also reported using millimetre scale ( $2 \mathrm{~mm}$ edge) particles of foamed polystyrene (FPS) coated with hydrophobic particles as the basis for liquid-marble type objects. ${ }^{98}$ An alternative type of composite liquid marble is one containing two immiscible liquids in distinct spatial arrangements, e.g. di-iodomethane and water, ${ }^{99}$ which can be re-shaped using an electric field. Composite liquid marbles can also be divided using a superhydrophobic scalpel. ${ }^{100}$ It has also been demonstrated that a liquid marble immersed in oil and subject to an electric field can distort and form a Taylor cone followed by jetting of a small drop. ${ }^{\mathbf{1 0 1}}$ If the liquid inside a marble is a ferrofluid it is possible to use a magnetic field to flatten the shape. ${ }^{\mathbf{1 0 2}}$ It is also possible to create oil-marbles contained within water-marbles as powdered oil-water emulsions. ${ }^{\mathbf{1 0 3}}$

Usually liquid marbles seek to minimise energy under the interaction of surface tension by adopting spherical shapes when small (height significantly less than the twice the capillary length) and gravitationally flattened puddle shapes when large. Using electric or magnetic fields, or introducing specific particle shapes, introduces other energy terms that alter the shape of the minimum energy configuration. For liquid metals using alloys of Gallium a mechanically deformed shape can be sustained after the removal of the deforming force due to the action of the oxide layer. Such shape retention ability for a water droplet appears to have been demonstrated using a sol-gel silica coated slide (and hydrophobic fumed silica) to shape a droplet and transfer particles to its surface, although the underlying mechanism appears unclear (Fig. 10a). ${ }^{\mathbf{1 0 4}}$

The particle encapsulation of ionic liquids into a liquid marble can be an intermediate step which allows polymerization of the entire ionic liquid surface area, and hence a complete polymer shell encapsulation. One approach it to use initiated chemical vapour deposition (iCVD), ${ }^{\mathbf{1 0 6}}$ whilst a simple alternative approach, motivated by its use in recovering fingerprint patterns, is to use fumed superglue. ${ }^{105}$ For iCVD to work successfully the energetics of the surfaces must favour the deposited polymer to spread over the core liquid. ${ }^{\mathbf{1 0 7}}$ It is also possible to change the morphology of the particle coating using exposure to solvent vapour to convert from a granular form to a thin film form of shell $^{\mathbf{1 0 8}}$ or via $\mathrm{pH}$ triggered membrane formation of self-assembling peptide units on the interior of the marble shell structure. ${ }^{109}$ Such changes may suppress evaporation of the core liquid and can increase the mechanical stability of the objects. Moreover, liquid marbles can be transformed into polymeric capsules containing water by exposure to solvent vapour and these can retain their shapes even after complete water loss and so produce rigid hollow polymeric capsules. ${ }^{\mathbf{1 1 0}}$ Edible fatty acid and tricylglycerol crystal based liquid marbles and capsules created by heating the liquid marbles to convert the fatty acid crystals to lipid shells have also been prepared. ${ }^{\mathbf{1 1 1}}$

It is interesting to speculate whether there may be ideas common to SLIPS surfaces in how a polymer encapsulation process might work. If we regard the particles forming the liquid marbles as a surface texture, polymer deposition onto a liquid marble is reminiscent of the need for an impregnating fluid to spread in (and over) a surface texture when forming a SLIPS surface. Similarly, given droplets on SLIPS surfaces can become cloaked in the impregnating fluid, coating a liquid marble might be possible simply by contacting the liquid marble to a suitable bath of impregnating fluid.

\subsection{Liquid marble impact}

Earlier studies of self-coating of droplets on loose hydrophobic particle surfaces, including extreme water repellent soil, ${ }^{\mathbf{1 1 2}}$ leading to liquid marble formation have continued with highspeed video of impact onto hydrophilic and hydrophobic glass spheres and soil. ${ }^{113}$ Formation of liquid marbles through impact of rain on hydrophobic soil may have implications for
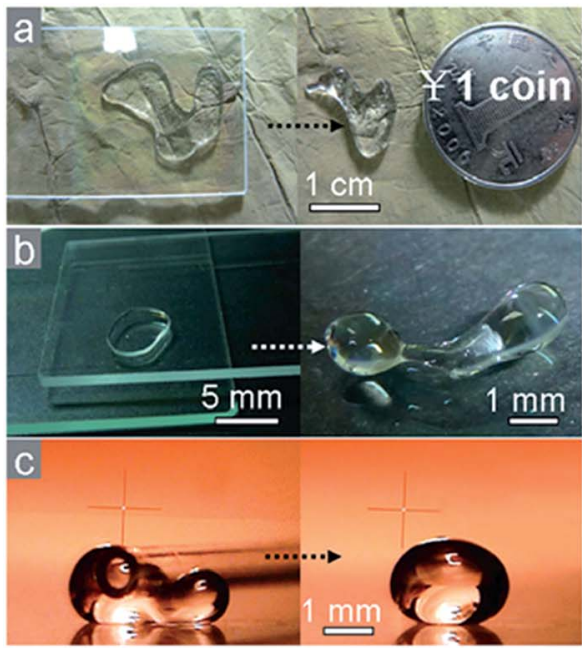

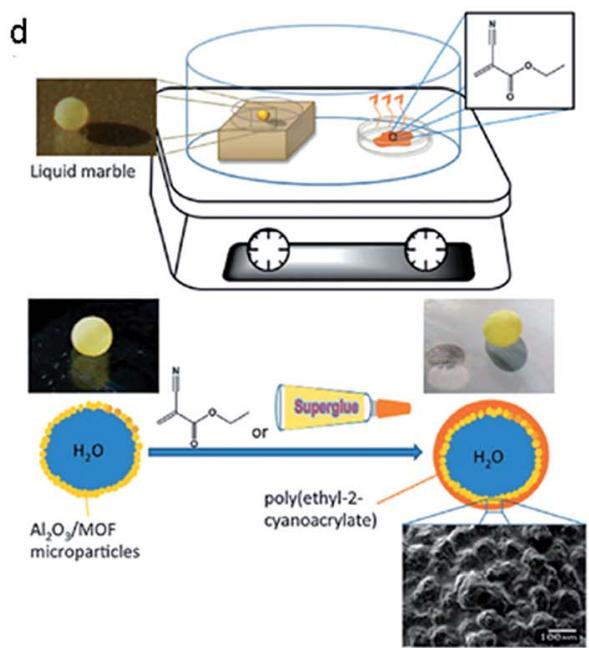

Fig. 10 (a)-(c) Deforming water drops using particle transfer by mechanical manipulation with sol-gel prepared hydrophobic silica particle coated glass slides and tubes (reproduced from ref. 104 with permission from The Royal Society of Chemistry). (d) Interfacial polymerization of ethyl-2-cyanoacrylate by vaporization and condensation on a liquid marble (i.e. "fingerprint fuming" process) (reproduced from ref. 105 with permission from The Royal Society of Chemistry). 
erosion. Impact formation of liquid marbles is discussed in detail by Eshtiaghi and Hapgood. ${ }^{114}$ Liquid marbles formed by gentle deposition and rolling of droplets on a bed of hydrophobic particles can be different to those formed from impact where attachment occurs during distortion of the droplet shape and internal flows due to the kinetic energy of the impact, see e.g. Whitby et al. ${ }^{48}$ It has also been reported that beyond a critical impact speed the resulting liquid marble is no longer spherical during rebound, but can take on a deformed cylindrical shape until it re-impacts the powder bed. ${ }^{\mathbf{1 1 5}}$

Three regimes of bouncing, sticking and rupture have been reported for impacting liquid marbles formed from relatively large (32 -159 $\mu \mathrm{m}$ diameter) glass spheres in a soft jamming layer state. ${ }^{\mathbf{1 1 6}}$ These authors also investigate to what extent liquid marbles impacting a smooth surface is similar to bare droplets impacting on micro-textured superhydrophobic surfaces and onto Leidenfrost surfaces. They discuss the effect of multi-particle layers requiring sufficient impact to cause "dejamming" in order for elastic behaviour to be observed. In a further study, Planchette et al. studied impact of droplets and armoured liquid marbles onto armoured puddles and deduced the threshold velocities for coalescence. ${ }^{117}$ Zang et al. also studied liquid marble impacts onto glass substrates and reported damping of shape oscillations during rebound. ${ }^{118}$ This latter report extracts dynamic surface tensions from the oscillation data and suggests a strong dependence on the hydrophobicity of the particles, which can be tuned to increase the dynamic surface tension and shorten impact contact time.

\section{Applications}

\subsection{Stimuli responsive liquid marbles}

In previous sections on magnetic particles and shaping shells we discussed the response of liquid marbles, including distortion and actuation, to electric and magnetic fields. We also described electrochemical and photochemical triggers for liquid metal marbles. In this section we focus on other types of external stimuli.

In designing stimuli responsive liquid marbles a common approach is to trigger liquid marble disintegration (a digital yes/ no response) when it is resting on an aqueous phase. An alternative is to trigger a colour change (an analogue range of values response). Recent work represents further development of principles reported in articles covered by our earlier Emerging Area paper. ${ }^{4}$ An example is the preparation of particles whose liquid-marble stabilising properties switch off at some value of $\mathrm{pH}$, thus allowing a critical change of the acidity/alkalinity of a supporting aqueous sub-phase to be detected. ${ }^{119-121}$ A procedure for the preparation of water "liquid marbles" taking advantage of the "salt responsive" character of the poly(ionic liquid)s for liquid marble stabilizers has also been reported. ${ }^{122}$ The principle of triggered disintegration of a liquid marble supported by a liquid sub-phase can be applied widely and has been demonstrated using sub-phase temperature ${ }^{\mathbf{1 2 3}}$ and exposure to near infrared ${ }^{\mathbf{6 8}}$ or ultraviolet light ${ }^{\mathbf{1 2 4}}$ irradiation. Gas-responsive particulate liquid marble stabilizers showing a stable to disintegration response when $\mathrm{H}_{2} \mathrm{O}$ vapour is replaced by $\mathrm{HCl}$ gas have been demonstrated. ${ }^{\mathbf{1 2 5}}$ Combining magnetic properties for actuation with stimuli responsiveness has been an objective of several authors. Zhang reported magnetic field responsive liquid marbles with destabilisation and rupture triggered by $\mathrm{pH}$ or ultraviolet light. ${ }^{53}$ Zhao et al. focused on multi-compartment liquid marbles, including structural colours that they suggested could be used for bar-coding applications. ${ }^{57} \mathrm{Xu}$ et al. have reported magnetic/non-magnetic semi-shell liquid marbles with triggered collapse due to either temperature or acid/base vapours. ${ }^{\mathbf{5 8}}$

\subsection{Miniature and micro-chemical reactors}

There has been a continuation of interest in using liquid marbles as self-contained reaction vessels taking advantage of the ability for a liquid to be simultaneously encapsulated and isolated from a substrate. By choosing a core liquid to be a $\mathrm{CuCl}_{2}$ solution an external stimulus can create a reaction inducing a quantitative colour change, rather than a yes/no disintegration response, related to the concentration of ammonia gas. ${ }^{\mathbf{1 2 6 , 1 2 7}}$ Ammonia gas has also been sensed via colour change using liquid marbles encapsulated with plasma treated hydrophobic cellulose particles and containing $\mathrm{CoCl}_{2}$ aqueous solutions. ${ }^{128}$ Reactions between mixtures of ammonia acetate, acetic acid and acetylacetone exposed to formaldehyde vapour have also been reported. ${ }^{129}$ Moreover, the use of powders of dry-water (or more precisely dry-solutions) allows improved quantitative measurement of colour due to the large numbers of individual liquid marbles.

Miniature reactors using superhydrophobic $\mathrm{Fe}_{3} \mathrm{O}_{4} / \mathrm{C}$ microsheets as encapsulating agents have been used for the synthesis of graphene-based nanocomposites. ${ }^{75}$ An interesting variation on the theme of miniature reactors is the deliberate use of the encapsulating particles to catalyse a reaction ("catalytic liquid marble reactor"), as demonstrated by Miao et al. using Ag nanowires for the rate-controllable catalytic reduction of encapsulated aqueous methylene blue in the presence of sodium borohydride $\left(\mathrm{NaBH}_{4}\right)$ (Fig. 11a). ${ }^{130}$ They suggest this as a system that provides support-free heterogeneous catalysis of small volume reactions for toxic and costly reactants and/or hazardous reactions where preliminary reactions involving small volume are preferred.

The idea of using the encapsulating particles as an active component in detection has also been developed with the concept of a "plasmonic liquid marble". ${ }^{131}$ This used metallic (Ag or $\mathrm{Au}$ ) nanoparticles with Surface Enhanced Raman Spectroscopy (SERS) to produce a substrate-less analytical platform to enable the simultaneous quantification and identification of target analytes (Fig. 11b). A variation on the idea of using two liquid marbles to hold separate liquids before coalescing them to initiate a reaction is to keep them as separate "cells", but electrically connect them via a conducting salt bridge to create a micro-electrochemical reactor (a "Daniell cell liquid marble"). ${ }^{132}$ This can generate an electric current or potential as the reaction control and monitoring mechanism.

The focus of reports on liquid marbles as reactors has generally been on their use as miniature reactors, but it is 
(A)
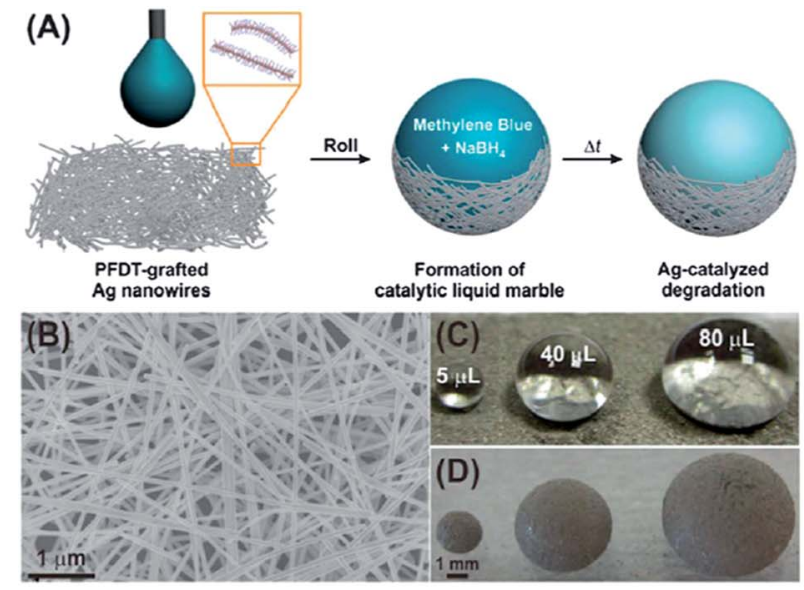

(E)

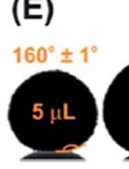

$\stackrel{\text { Roll }}{\longrightarrow}$
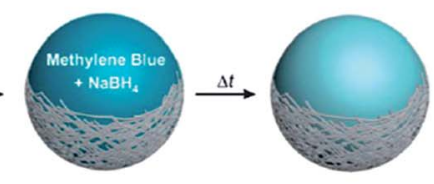

Formation of Formation of
catalytic liquid marble

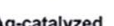
Ag-catalyzed
degradation

(F)
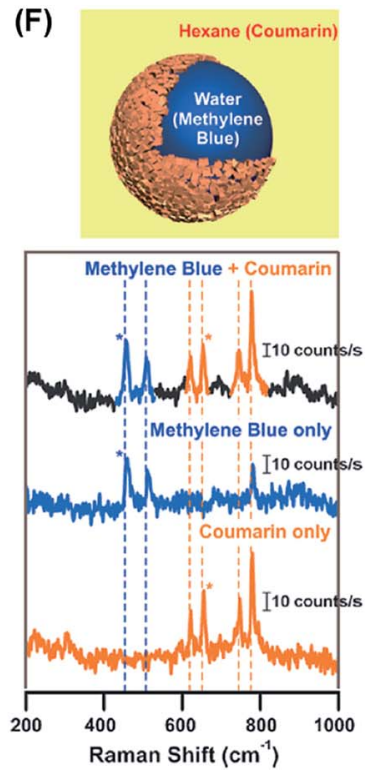

Fig. 11 (A)-(E) Ag nanowire-based catalytic liquid marbles (reproduced from ref. 130 with permission from The Royal Society of Chemistry). (F) Plasmonic liquid marble formed using metallic nanoparticles for substrate-less surface enhanced Raman spectroscopy (reproduced from ref. 131. Copright@ 2014 WILEY VCH Verlag GmbH \& Co. KGaA, Weinheim).

entirely plausible that the volumes involved could be scaled up to macro-reactors using liquid puddles. These would have maximum height of twice the capillary length $(\sim 5.46 \mathrm{~mm}$ for water), but could have a much larger lateral extent. Reactors could have micro- or macro-scale magnetic bar stirrers incorporated. The transition from a liquid marble having minimum surface area-to-volume ratio to a liquid puddle with a large surface area-to-volume ratio might be of value when using the containing particles as catalysts (i.e. a catalytic liquid puddle). Similarly, a larger surface area may be of value to enable SERS (i.e. plasmonic liquid puddles) or in other applications where the encapsulating particles have an active role in enabling or detecting reactions. It is also possible to imagine a similar approach to Su et al. who used an aqueous drop as the miniature reactor, but resting on a superhydrophobic surface immersed in silicone oil in order to apply heat to drive a reaction and remove evaporation. ${ }^{133}$ The analogous case would use a liquid marble immersed in a suitable liquid.

\subsection{Miniature and micro-bioreactors}

A natural extension of the use of a liquid marble as a microreactor is into the biological domain for use in biological reactions and diagnostic assays. The effectiveness of this broad principle was demonstrated for $\mathrm{ABO}$ and $\mathrm{Rh}$ blood typing by creating blood liquid marbles, injecting them with antibodies to test for haemagglutination and using a simple colour change as a visual indication of reaction (Fig. 12a). ${ }^{134}$ The authors suggested four advantages, (i) small amounts of samples and reagents required, (ii) reduced biohazard due to isolation from surfaces, (iii) simple control of reagents by coalescence or injection into marble, and (iv) inexpensive and hence disposable. Arbatan et al. have also reported the use of liquid marbles for cell culture. ${ }^{135}$ They note that to culture cancer cell spheroids (CCSs) in vitro in a form that may reflect the in vivo physiology of tumors more realistically than $2 \mathrm{D}$ cell cultures, multi-well trays are often inverted to create systems of multiple hanging drops of cell suspensions. A liquid marble with non-adhesive particles (for the cells) provides a realistic 3D volume and has maximum potential contact for aggregation due to the maximum volumeto-surface area property of the marble (Fig. $12 \mathrm{~b}$ and c). Related reports based on the liquid marbles ability to remove the problem of cell adhesion to the base of a cell culture dish and being able to keep cells in suspension have shown their use as platforms for the culturing of embryoid bodies (EBs) from embryonic stem cells (ES cells). ${ }^{136}$ In complementary work, Oliveira et al. showed that liquid marbles could be used for high-throughput drug screening requiring anchorage-dependent cells by dispersing suitable particles within the core liquid of the liquid marble (Fig. 12d). ${ }^{137}$ In the context of bioreactors and perfusion for long term cell culture, but also more general microfluidic requirements, in-flow and outflow of liquid into liquid marbles and its effect on marble stability has been studied ${ }^{138}$ Taking advantage of the gas permeable nature of the shell liquid marbles have also been used for culturing aerobic microorganisms. ${ }^{139}$ It is also interesting to note a recent report that flips the idea from using liquid marbles as reactors to using them for cryopreservation of mammalian cell lines. ${ }^{140}$

In applications using the permeability to gases of the liquid marble surface the use of larger liquid puddles would increase the overall surface area-to-volume ratio for gas exchange. In considering the current evolution in applications of liquid marbles towards biology it is interesting to consider whether there could be applications in DNA/RNA/protein microarray technology. ${ }^{141}$ Currently, methods of producing microarrays include robotic spotting, lithography and bead-based assembly. 

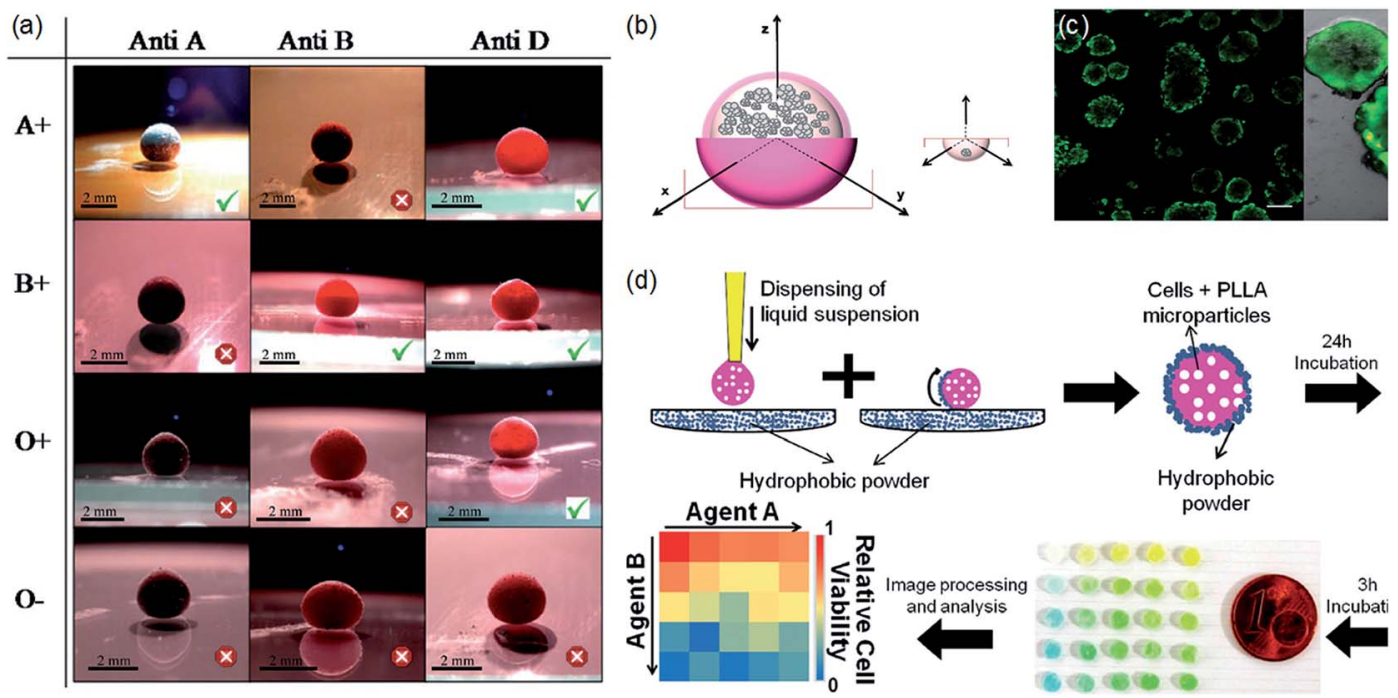

Fig. 12 (a) Blood typing by injecting antibodies into blood marbles (reproduced from ref. 134. (Copyright@ 2012 WILEY VCH Verlag GmbH \& Co. KGaA, Weinheim)). (b) Formation of cancer cell spheroid formation in a liquid marble (variable size marble) versus handing drop (fixed maximum drop size). (c) Three dimensional aggregation of Hep G2 cells ((b) and (c) reproduced from ref. 135. (Copyright@ 2012 WILEY VCH Verlag GmbH \& Co. KGaA, Weinheim)). (d) High throughput drug screening (reproduced from ref. 137. Copyright@ 2014 WILEY VCH Verlag GmbH \& Co. KGaA, Weinheim).

One can speculate that robotic spotting could be combined with hydrophobic particles contained within dimples. Alternatively particles used to encapsulate liquid marbles or dispersed within a liquid marble could form part of a microarray approach.

\subsection{Electrostatic-based applications}

Liquid marbles with conductive/ionic cores have been considered as possible electrostatic-based devices for harvesting energy from low frequency vibrations on a charged thin-film electret. ${ }^{\mathbf{1 4 2}}$ Electrostatics has also been considered to induce particle coating of pendant droplets and it has been suggested that complex multi-layered agglomerates produced by this method could have potential industrial, pharmaceutical, environmental, and biological applications. ${ }^{143}$

\subsection{Patents/patent applications}

Identifying liquid marble and dry water related patents and patent applications is far from straightforward. In our previous Emerging Area article ${ }^{4}$ we noted a number of patents including a 1968 Degussa patent on dry water. In updating our literature review, we identified a 2002 patent "Particulate encapsulation of liquid beads" with a priority date from 2000, which contains many ideas familiar from liquid marbles. ${ }^{\mathbf{1 4 4}}$ Moreover, there are two patents from 1998 and 2000 relating to hydrophobic particle coated droplets as chambers for chemical and biological reactions. ${ }^{\mathbf{1 4 5 , 1 4 6}}$ All of these patents pre-date the current academic literature on liquid marbles.

Recent academic-based work has resulted in accompanying patents on the ideas of stimulus responsive liquid marbles, ${ }^{\mathbf{1 4 7}}$ clathrates for gas storage, ${ }^{\mathbf{1 4 8}}$ methods for making nanofibrillated cellulose particles ${ }^{71}$ and methods for making emulsions. ${ }^{\mathbf{1 4 9}}$ Other recent industrial-based patents that we identified tended to be dry water related for cosmetics of one type or another. These included ones that changed colour on application, ${ }^{150}$ contain glycerol for skin cosmetics, ${ }^{151}$ and glycerol and other polyols for hair cosmetics, ${ }^{\mathbf{1 5 2}}$ and preparation and production processes. ${ }^{153,154}$

\section{Summary}

The field of liquid marbles has advanced substantially over the last few years from basic principles and studies of them as exotic objects to detailed methods allowing tailored objects for specific purposes. At the fundamental level there are reports that lack information on the surface state of particles (singlelayer or multi-layer, and liquid-like or solid-like packing), which is needed to understand heat and mass transfer and impact properties. At the application level, previous potential is now being realized, such as in their use as sensors and as selfassembling, self-healing miniature chemical reactors, including with particles as catalytic sites. The biological applications are fascinating with blood typing, and cell and microorganism cultures using the ability to isolate liquids from the substrate and create three-dimensional cultures. A novel direction is the use of liquid metal marbles, and the suggestion that surface oxides could be considered as surfactants for liquid metals. This also highlights questions around how the liquid marble shape can be switched and controlled and why there is relatively little focus on the use of liquid puddles macro-reactors where surface-area-to-volume ratio is maximised rather than minimised. It is also interesting to consider to what extent the idea of a liquid marble with added texture that is conformable to a droplet shape and lifts/insulates it from a substrate can leverage ideas from superhydrophobic surfaces, capillary origami, SLIPS 
surfaces and Leidenfrost droplets. We have speculated on whether this can lead to liquid-infused liquid marble surfaces, and new kinds of particle stabilized interfaces, emulsions and compound droplets. Finally, progress in biological applications and the ability to create multiple small distinct objects suggests there may be potential for new types of microarrays.

\section{Acknowledgements}

The authors' acknowledge financial assistance from the U.K. Engineering and Physical Sciences Research Council (Grant nos EP/H000704/1, EP/E063489/1, EP/L026341/1 and EP/L026899/1).

\section{References}

1 P. Aussillous and D. Quéré, Nature, 2001, 411, 924-927.

2 P. Aussillous and D. Quéré, Proc. R. Soc. A, 2006, 462, 973999.

3 G. McHale, Langmuir, 2009, 25, 7185-7187.

4 G. McHale and M. I. Newton, Soft Matter, 2011, 7, 54735481.

5 N. Pike, D. Richard, W. Foster and L. Mahadevan, Proc. $R$. Soc. B, 2002, 269, 1211-1215.

6 E. Bormashenko, Curr. Opin. Colloid Interface Sci., 2011, 16, 266-271.

7 E. Bormashenko, Soft Matter, 2012, 8, 11018-11021.

8 L. Mahadevan and Y. Pomeau, Phys. Fluids, 1999, 11, 24492453.

9 P. Aussillous and D. Quéré, J. Fluid Mech., 2004, 512, 133151.

10 B. P. Binks and R. Murakami, Nat. Mater., 2006, 5, 865-869. 11 D. Quéré, Annu. Rev. Mater. Res., 2008, 38, 71-99.

12 C. Py, P. Reverdy, L. Doppler, J. Bico, B. Roman and C. Baroud, Phys. Rev. Lett., 2007, 98, 2-5.

13 T.-S. Wong, S. H. Kang, S. K. Y. Tang, E. J. Smythe, B. D. Hatton, A. Grinthal and J. Aizenberg, Nature, 2011, 477, 443-447.

14 A.-L. Biance, C. Clanet and D. Quéré, Phys. Fluids, 2003, 15, 1632-1637.

15 P. Roach, N. J. Shirtcliffe and M. I. Newton, Soft Matter, 2008, 4, 224-240.

16 N. J. Shirtcliffe, G. McHale, S. Atherton and M. I. Newton, Adv. Colloid Interface Sci., 2010, 161, 124-138.

17 M. Callies and D. Quéré, Soft Matter, 2005, 1, 55-61.

18 G. McHale, D. L. Herbertson, S. J. Elliott, N. J. Shirtcliffe and M. I. Newton, Langmuir, 2007, 23, 918-924.

19 J. Bico, B. Roman, L. Moulin and A. Boudaoud, Nature, 2004, 432, 690.

20 G. McHale, Langmuir, 2009, 25, 7185-7187.

21 G. McHale, M. I. Newton, N. J. Shirtcliffe and N. R. Geraldi, Beilstein J. Nanotechnol., 2011, 2, 145-151.

22 N. R. Geraldi, F. F. Ouali, R. H. Morris, G. McHale and M. I. Newton, Appl. Phys. Lett., 2013, 102, 214104.

23 L. Gao and T. J. McCarthy, Langmuir, 2008, 24, 9183-9188. 24 J. D. Smith, R. Dhiman, S. Anand, E. Reza-Garduno, R. E. Cohen, G. H. McKinley and K. K. Varanasi, Soft Matter, 2013, 9, 1772-1780.
25 D. Quéré, Annu. Rev. Fluid Mech., 2013, 45, 197-215.

26 M. D. Doganci, B. U. Sesli, H. Y. Erbil, B. P. Binks and I. E. Salama, Colloids Surf., A, 2011, 384, 417-426.

27 H. Y. Erbil, Adv. Colloid Interface Sci., 2012, 170, 67-86.

28 B. Laborie, F. Lachaussée, E. Lorenceau and F. Rouyer, Soft Matter, 2013, 9, 4822-4830.

29 C. Aberle, M. Lewis, G. Yu, N. Lei and J. Xu, Soft Matter, 2011, 7, 11314-11318.

30 I. U. Vakarelski, N. a. Patankar, J. O. Marston, D. Y. C. Chan and S. T. Thoroddsen, Nature, 2012, 489, 274-277.

31 K. Saleh, L. Forny, P. Guigon and I. Pezron, Chem. Eng. Res. Des., 2011, 89, 537-544.

32 K. Rykaczewski, J. Chinn, M. L. Walker, J. H. J. Scott, A. Chinn and W. Jones, ACS Nano, 2011, 5, 9746-9754.

33 P. S. Bhosale and M. V Panchagnula, Langmuir, 2012, 28, 14860-14866.

34 A. Hashmi, A. Strauss and J. Xu, Langmuir, 2012, 28, 1032410328.

35 D. Zang, K. Lin, W. Wang, Y. Gu, Y. Zhang, X. Geng and B. P. Binks, Soft Matter, 2014, 10, 1309-1314.

36 L. Gao and T. J. McCarthy, Langmuir, 2007, 23, 1044510447.

37 K. Shirato and M. Satoh, Soft Matter, 2011, 7, 7191-7193.

38 J. Yang, Z.-Z. Zhang, X.-H. Men and X.-H. Xu, J. Dispersion Sci. Technol., 2011, 32, 485-489.

39 B. P. Binks and A. T. Tyowua, Soft Matter, 2013, 9, 834-845.

40 B. P. Binks, T. Sekine and A. T. Tyowua, Soft Matter, 2014, 10, 578-589.

41 P. D. I. Fletcher and B. L. Holt, Langmuir, 2011, 27, 1286912876.

42 B. P. Binks, P. D. I. Fletcher, M. A. Thompson and R. P. Elliott, Langmuir, 2013, 29, 5723-5733.

43 A. K. F. Dyab and H. N. Al-Haque, RSC Adv., 2013, 3, 1310113105.

44 E. Bormashenko, R. Pogreb and A. Musin, J. Colloid Interface Sci., 2012, 366, 196-199.

45 D. Matsukuma, H. Watanabe, H. Yamaguchi and A. Takahara, Langmuir, 2011, 27, 1269-1274.

46 C. A. E. Hamlett, N. J. Shirtcliffe, G. McHale, S. Ahn, R. Bryant, S. H. Doerr and M. I. Newton, Environ. Sci. Technol., 2011, 45, 9666-9670.

47 E. Bormashenko, R. Balter and D. Aurbach, J. Colloid Interface Sci., 2012, 384, 157-161.

48 C. P. Whitby, X. Bian and R. Sedev, Soft Matter, 2012, 8, 11336-11342.

49 C. P. Whitby, X. Bian and R. Sedev, Colloids Surf., A, 2013, 436, 639-646.

50 N. J. Shirtcliffe, G. McHale, M. I. Newton, F. B. Pyatt and S. H. Doerr, Appl. Phys. Lett., 2006, 89, 094101.

51 N. Tippkötter, H. Al-Kaidy, S. Wollny and R. Ulber, Chem. Ing. Tech., 2013, 85, 76-86.

52 Y. Zhao, Z. Xu, M. Parhizkar, J. Fang, X. Wang and T. Lin, Microfluid. Nanofluid., 2012, 13, 555-564.

53 L. Zhang, D. Cha and P. Wang, Adv. Mater., 2012, 24, 47564760.

54 S. Zhang, Y. Zhang, Y. Wang, S. Liu and Y. Deng, Phys. Chem. Chem. Phys., 2012, 14, 5132-5138. 
55 Y. Hu, H. Jiang, J. Liu, Y. Li, X. Hou and C. Li, RSC Adv., 2014, 4, 3162-3164.

56 Y. Li, Y. Hu, H. Jiang and C. Li, Nanoscale, 2013, 5, 53605367.

57 Y. Zhao, H. Gu, Z. Xie, H. C. Shum, B. Wang and Z. Gu, J. Am. Chem. Soc., 2013, 135, 54-57.

58 Z. Xu, Y. Zhao, L. Dai and T. Lin, Part. Part. Syst. Charact., 2014, 31, 839-842.

59 K. S. Khalil, S. R. Mahmoudi, N. Abu-dheir and K. K. Varanasi, Appl. Phys. Lett., 2014, 105, 041604.

60 D. Quéré, J. M. Di Meglio and F. Brochard-Wyart, Rev. Phys. Appl., 1988, 23, 1023-1030.

61 B. P. Binks, A. N. Boa, M. A. Kibble, G. Mackenzie and A. Rocher, Soft Matter, 2011, 7, 4017-4024.

62 A. Yildirim, H. Budunoglu, B. Daglar, H. Deniz and M. Bayindir, ACS Appl. Mater. Interfaces, 2011, 3, 1804-1808.

63 M. T. Gokmen, B. Dereli, B. G. De Geest and F. E. Du Prez, Part. Part. Syst. Charact., 2013, 30, 438-444.

64 Y. Lai, Y. Tang, J. Huang, H. Wang, H. Li, D. Gong, X. Ji, J. Gong, C. Lin, L. Sun and Z. Chen, Soft Matter, 2011, 7, 6313-6319.

65 G. M. Walker, P. McEleney, A. H. Al-Muhtaseb and S. E. J. Bell, Chem. Eng. J., 2013, 228, 984-992.

66 L. Zhang, J. Wu, Y. Wang, Y. Long, N. Zhao and J. Xu, J. Am. Chem. Soc., 2012, 134, 9879-9881.

67 W. Liang, G. Wang, B. Wang, Y. Zhang and Z. Guo, Acta Chim. Sin., 2013, 71, 639-643.

68 K. Nakai, H. Nakagawa, K. Kuroda, S. Fujii, Y. Nakamura and S. Yusa, Chem. Lett., 2013, 42, 719-721.

69 H. Wu, H. Watanabe, W. Ma, A. Fujimoto, T. Higuchi, K. Uesugi, A. Takeuchi, Y. Suzuki, H. Jinnai and A. Takahara, Langmuir, 2013, 29, 14971-14975.

70 H. Mertaniemi, A. Laukkanen, J.-E. Teirfolk, O. Ikkala and R. H. a. Ras, RSC Adv., 2012, 2, 2882-2886.

71 A. Laukkanen, J.-E. Teirfolk, O. Ikkala, R. Ras and H. Mertaniemi, Hydrophobic coating and a method for producing hy, US Pat. App. 20140130710, 2014.

72 E. Mele, I. S. Bayer, G. Nanni, J. A. Heredia-Guerrero, R. Ruffilli, F. Ayadi, L. Marini, R. Cingolani and A. Athanassiou, Langmuir, 2014, 30, 2896-2902.

73 Y. Xue, Y. Liu, F. Lu, J. Qu, H. Chen and L. Dai, J. Phys. Chem. Lett., 2012, 3, 1607-1612.

74 H. Hu, C. C. K. Allan, J. Li, Y. Kong, X. Wang, J. H. Xin and H. Hu, Nano Res., 2014, 7, 418-433.

75 Y. Chu, Z. Wang and Q. Pan, ACS Appl. Mater. Interfaces, 2014, 6, 8378-8386.

76 A. F. Mejia, A. Diaz, S. Pullela, Y.-W. Chang, M. Simonetty, C. Carpenter, J. D. Batteas, M. S. Mannan, A. Clearfield and Z. Cheng, Soft Matter, 2012, 8, 10245-10253.

77 M. D. Dickey, ACS Appl. Mater. Interfaces, 2014, 6, 1836918379.

78 C. Ladd, J.-H. So, J. Muth and M. D. Dickey, Adv. Mater., 2013, 25, 5081-5085.

79 E. Palleau, S. Reece, S. C. Desai, M. E. Smith and M. D. Dickey, Adv. Mater., 2013, 25, 1589-1592.

80 M. R. Khan, C. B. Eaker, E. F. Bowden and M. D. Dickey, Proc. Natl. Acad. Sci. U. S. A., 2014, 111, 14047-14051.
81 V. Sivan, S.-Y. Tang, A. P. O'Mullane, P. Petersen, N. Eshtiaghi, K. Kalantar-zadeh and A. Mitchell, Adv. Funct. Mater., 2013, 23, 144-152.

82 X. Tang, S.-Y. Tang, V. Sivan, W. Zhang, A. Mitchell, K. Kalantar-zadeh and K. Khoshmanesh, Appl. Phys. Lett., 2013, 103, 174104.

83 S.-Y. Tang, V. Sivan, K. Khoshmanesh, A. P. O'Mullane, X. Tang, B. Gol, N. Eshtiaghi, F. Lieder, P. Petersen, A. Mitchell and K. Kalantar-zadeh, Nanoscale, 2013, 5, 5949-5957.

84 D. Vella and L. Mahadevan, Am. J. Phys., 2005, 73, 817-825. 85 P. A. Kralchevsky and K. Nagayama, Adv. Colloid Interface Sci., 2000, 85, 145-192.

86 A. B. Subramaniam, M. Abkarian and H. A. Stone, Nat. Mater., 2005, 4, 553-556.

87 G. Lagubeau, A. Rescaglio and F. Melo, Phys. Rev. E: Stat., Nonlinear, Soft Matter Phys., 2014, 90, 030201.

88 A. B. Subramaniam, M. Abkarian, L. Mahadevan and H. A. Stone, Nature, 2005, 438, 930.

89 C. Planchette, E. Lorenceau and A.-L. Biance, Soft Matter, 2012, 8, 2444-2451.

90 D. Vella, P. Aussillous and L. Mahadevan, Europhys. Lett., 2004, 68, 212-218.

91 H. N. Dixit and G. M. Homsy, J. Fluid Mech., 2013, 732, 5-28. 92 E. Bormashenko, A. Musin, G. Whyman, Z. Barkay, A. Starostin, V. Valtsifer and V. Strelnikov, Colloids Surf., A, 2013, 425, 15-23.

93 S. Ogawa, H. Watanabe, L. Wang, H. Jinnai, T. J. McCarthy and A. Takahara, Langmuir, 2014, 30, 9071-9075.

94 T. Arbatan and W. Shen, Langmuir, 2011, 27, 12923-12929. 95 U. Cengiz and H. Y. Erbil, Soft Matter, 2013, 9, 8980-8991.

96 M. Abkarian, S. Protière, J. M. Aristoff and H. A. Stone, Nat. Commun., 2013, 4, 1895.

97 E. Bormashenko, Y. Bormashenko, R. Pogreb and O. Gendelman, Langmuir, 2011, 27, 7-10.

98 E. Bormashenko, R. Balter, H. Aharoni and D. Aurbach, J. Colloid Interface Sci., 2014, 417, 206-209.

99 E. Bormashenko, R. Pogreb, R. Balter, O. Gendelman and D. Aurbach, Appl. Phys. Lett., 2012, 100, 151601.

100 E. Bormashenko and Y. Bormashenko, Langmuir, 2011, 27, 3266-3270.

101 E. Bormashenko, R. Pogreb, G. Whyman and A. Musin, Colloid Polym. Sci., 2013, 291, 1535-1539.

102 N. Nguyen, Langmuir, 2013, 29, 13982-13989.

103 R. Murakami, H. Moriyama, T. Noguchi, M. Yamamoto and B. P. Binks, Langmuir, 2014, 30, 496-500.

104 X. Li and J. Shen, Chem. Commun., 2013, 49, 10016-10018. 105 J. M. Chin, M. R. Reithofer, T. T. Y. Tan, A. G. Menon, E. Y. Chen, C. A. Chow, A. T. S. Hor and J. Xu, Chem. Commun., 2013, 49, 493-495.

106 L. C. Bradley and M. Gupta, Langmuir, 2012, 28, 1027610280.

107 P. D. Haller, L. C. Bradley and M. Gupta, Langmuir, 2013, 29, 11640-11645.

108 D. Matsukuma, H. Watanabe, M. Minn, A. Fujimoto, T. Shinohara, H. Jinnai and A. Takahara, RSC Adv., 2013, 3, 7862-7866. 
109 H.-G. Braun and A. Z. Cardoso, Colloids Surf., B, 2012, 97, 43-50.

110 K. Ueno, S. Hamasaki, E. J. Wanless, Y. Nakamura and S. Fujii, Langmuir, 2014, 30, 3051-3059.

111 Y. Kawamura, H. Mayama and Y. Nonomura, J. Oleo Sci., 2012, 61, 477-482.

112 G. McHale, N. J. Shirtcliffe, M. I. Newton, F. B. Pyatt and S. H. Doerr, Appl. Phys. Lett., 2007, 90, 054110.

113 S. Ahn, S. H. Doerr, P. Douglas, R. Bryant, C. A. E. Hamlett, G. McHale, M. I. Newton and N. J. Shirtcliffe, Earth Surf. Processes Landforms, 2013, 38, 1225-1233.

114 N. Eshtiaghi and K. P. Hapgood, Powder Technol., 2012, 223, 65-76.

115 J. O. Marston, Y. Zhu, I. U. Vakarelski and S. T. Thoroddsen, Powder Technol., 2012, 228, 424-428.

116 C. Planchette, A.-L. Biance and E. Lorenceau, Europhys. Lett., 2012, 97, 14003.

117 C. Planchette, A.-L. Biance, O. Pitois and E. Lorenceau, Phys. Fluids, 2013, 25, 042104.

118 D. Zang, Z. Chen, Y. Zhang, K. Lin, X. Geng and B. P. Binks, Soft Matter, 2013, 9, 5067-5073.

119 S. Fujii, M. Suzaki, S. P. Armes, D. Dupin, S. Hamasaki, K. Aono and Y. Nakamura, Langmuir, 2011, 27, 8067-8074.

120 D. Dupin, K. L. Thompson and S. P. Armes, Soft Matter, 2011, 7, 6797-6800.

121 M. Inoue, S. Fujii, Y. Nakamura, Y. Iwasaki and S. Yusa, Polym. J., 2011, 43, 778-784.

122 A. M. Fernandes, R. Gracia, G. P. Leal, M. Paulis and D. Mecerreyes, Polymer, 2014, 55, 3397-3403.

123 S. Yusa, M. Morihara, K. Nakai, S. Fujii, Y. Nakamura, A. Maruyama and N. Shimada, Polym. J., 2014, 46, 145-148.

124 K. Nakai, S. Fujii, Y. Nakamura and S. Yusa, Chem. Lett., 2013, 42, 586-588.

125 S. Fujii, K. Aono, M. Suzaki, S. Hamasaki, S. Yusa and Y. Nakamura, Macromolecules, 2012, 45, 2863-2873.

126 M. Hu, M. Tian, J. He and Y. He, Colloids Surf., A, 2012, 414, 216-219.

127 K. Matsubara, M. Danno, M. Inoue, H. Nishizawa, Y. Honda and T. Abe, Surf. Coat. Technol., 2013, 236, 269-273.

128 T. Ihara and Y. Iriyama, J. Photopolym. Sci. Technol., 2011, 24, 435-440.

129 E. Bormashenko, R. Balter and D. Aurbach, Int. J. Chem. React. Eng., 2011, 9, 1542-6580.

130 Y.-E. Miao, H. K. Lee, W. S. Chew, I. Y. Phang, T. Liu and X. Y. Ling, Chem. Commun., 2014, 50, 5923-5926.

131 H. K. Lee, Y. H. Lee, I. Y. Phang, J. Wei, Y.-E. Miao, T. Liu and X. Y. Ling, Angew. Chem. Int. Ed., 2014, 53, 5054-5058.

132 M. Li, J. Tian, L. Li, A. Liu and W. Shen, Chem. Eng. Sci., 2013, 97, 337-343.
133 B. Su, S. Wang, Y. Song and L. Jiang, Soft Matter, 2012, 8, 631-635.

134 T. Arbatan, L. Li, J. Tian and W. Shen, Adv. Healthcare Mater., 2012, 1, 80-83.

135 T. Arbatan, A. Al-Abboodi, F. Sarvi, P. P. Y. Chan and W. Shen, Adv. Healthcare Mater., 2012, 1, 467-469.

136 F. Sarvi, T. Arbatan, P. P. Y. Chan and W. Shen, RSC Adv., 2013, 3, 14501-14508.

137 N. M. Oliveira, C. R. Correia, R. L. Reis and J. F. Mano, Adv. Healthcare Mater., 2014, 1-7.

138 A. Bajwa, Y. Xu, A. Hashmi, M. Leong, L. Ho and J. Xu, Soft Matter, 2012, 8, 11604-11608.

139 J. Tian, N. Fu, X. D. Chen and W. Shen, Colloids Surf., B, 2013, 106, 187-190.

140 M. C. Serrano, S. Nardecchia, M. C. Gutiérrez, M. L. Ferrer and F. del Monte, ACS Appl. Mater. Interfaces, 2015, 150204081729006.

141 G. McHale, Analyst, 2007, 132, 192-195.

142 Z. Yang, E. Halvorsen and T. Dong, J. Microelectromech. Syst., 2014, 23, 315-323.

143 K. R. Liyanaarachchi, P. M. Ireland, G. B. Webber and K. P. Galvin, Appl. Phys. Lett., 2013, 103, 054105.

144 M. A. Hamer, J. J. Marti and W. A. Hendrickson, Particulate encapsulation of liquid beads, US Pat., 6,413,548, 2002.

145 A. K. Shukla, Droplet chemical reaction chamber, US Pat., 5,773,238, 1998.

146 A. M. Shukla, A. K. Shukla and M. M. Shukla, Hydrophobic particle-coated aqueous droplet react, US Pat., 6,114,153, 2000.

147 S. P. Armes and D. Dupin, Liquid Composite Materials, World Pat. App. WO2010092191, 2010.

148 A. Cooper, D. Adams, C. Bray and W. Wang, Clathrates for gas storage, US Pat. App. 20110185623, 2011.

149 E. Bormashenko, Emulsions and methods of making emulsions, US Pat. App. 20140350168, 2014.

150 T. Zamyatin, I. Sandewicz, J. Russ and S. Jabush, Dry water cosmetic compositions that change color, US Pat. App. 20070218024, 2007.

151 H. Riedemann and J. Münzenberg, Core-shell particles with a high content of glyce, US Pat. App. 20120315312, 2012.

152 T. Knappe and A. Henschel, Powdery hair cosmetics, US Pat. App. 20140302106, 2014.

153 H. Pitsch, J. Piroth, T. Riedemann, H. Riedemann, A. Gray and F. D. Huenig, Dry liquids, and processes for their preparation, US Pat. App. 20090202835, 2009.

154 T. Oka, N. Koga, E. Takasu and T. Yanaki, Production method of dry water, US Pat. App. 20110165323, 2011. 